\title{
Low-coverage surface diffusion in complex periodic energy landscapes: Analytical solution for systems with symmetric hops and application to intercalation in topological insulators
}

\author{
Miguel A. Gosálvez, ${ }^{1,2,3,{ }^{*}}$ Mikhail M. Otrokov, ${ }^{2,4,5}$ Nestor Ferrando, ${ }^{3,2}$ Anastasia G. Ryabishchenkova, ${ }^{4}$ Andres Ayuela, ${ }^{3,2}$ \\ Pedro M. Echenique, ${ }^{1,2,3}$ and Evgueni V. Chulkov ${ }^{1,2,3,5}$ \\ ${ }^{1}$ Department of Materials Physics, University of the Basque Country UPV/EHU, 20018 Donostia-San Sebastian, Spain \\ ${ }^{2}$ Donostia International Physics Center (DIPC), 20018 Donostia-San Sebastian, Spain \\ ${ }^{3}$ Centro de Física de Materiales (CFM), Materials Physics Center (MPC), Centro Mixto CSIC-UPV/EHU, \\ 20018 Donostia-San Sebastian, Spain \\ ${ }^{4}$ Tomsk State University, 634050 Tomsk, Russia \\ ${ }^{5}$ Saint Petersburg State University, 198504 Saint Petersburg, Russia
}

(Received 1 September 2015; revised manuscript received 25 January 2016; published 18 February 2016)

\begin{abstract}
This is the first of two papers that introduce a general expression for the tracer diffusivity in complex, periodic energy landscapes with $M$ distinct hop rates in one-, two-, and three-dimensional diluted systems (low-coverage, single-tracer limit). The present report focuses on the analysis of diffusion in systems where the end sites of the hops are located symmetrically with respect to the hop origins (symmetric hops), as encountered in many ideal surfaces and bulk materials. For diffusion in two dimensions, a number of formulas are presented for complex combinations of the different hops in systems with triangular, rectangular, and square symmetry. The formulas provide values in excellent agreement with kinetic Monte Carlo simulations, concluding that the diffusion coefficient can be directly determined from the proposed expressions without performing the simulations. Based on the diffusion barriers obtained from first-principles calculations and a physically meaningful estimate of the attempt frequencies, the proposed formulas are used to analyze the diffusion of $\mathrm{Cu}, \mathrm{Ag}$, and $\mathrm{Rb}$ adatoms on the surface and within the van der Waals (vdW) gap of a model topological insulator, $\mathrm{Bi}_{2} \mathrm{Se}_{3}$. Considering the possibility of adsorbate intercalation from the terraces to the vdW gaps at morphological steps, we infer that, at low coverage and room temperature, (i) a majority of the $\mathrm{Rb}$ atoms bounce back at the steps and remain on the terraces, (ii) $\mathrm{Cu}$ atoms mostly intercalate into the $\mathrm{vdW}$ gap, the remaining fraction staying at the steps, and (iii) $\mathrm{Ag}$ atoms essentially accumulate at the steps and gradually intercalate into the vdW gap. These conclusions are in good qualitative agreement with previous experiments. The companion report (M. A. Gosálvez et al., Phys. Rev. B, submitted] extends the present study to the description of systems that contain asymmetric hops.
\end{abstract}

DOI: 10.1103/PhysRevB.93.075429

\section{INTRODUCTION}

The diffusion of atoms and molecules on crystalline surfaces is fundamental to several technologies [1-5]. These include heterogeneous catalysis for mass production of essential compounds in the chemical, food, and energy industries [6], as well as growth of thin films for the fabrication of semiconductor devices and novel two-dimensional (2D) materials, such as graphene [7-9]. Planar synthesis technologies, such as chemical vapor deposition, where surface diffusion plays a key role, are currently attracting increasing attention as an alternative to supply a complete, new generation of atom-thick materials, including semimetals (graphene, $\mathrm{NiTe}_{2}$, $\mathrm{VSe}_{2}$, etc.) [7,8,10-12], semiconductors $\left(\mathrm{WS}_{2}, \mathrm{WSe}_{2}, \mathrm{MoS}_{2}\right.$, $\mathrm{MoSe}_{2}, \mathrm{MoTe}_{2}, \mathrm{TaS}_{2}, \mathrm{RhTe}_{2}, \mathrm{PdTe}_{2}$, etc.) [10,13-15], insulators (hexagonal-BN, $\mathrm{HfS}_{2}$, etc.) $[13,16,17]$, superconductors $\left(\mathrm{NbS}_{2}, \mathrm{NbSe}_{2}, \mathrm{NbTe}_{2}, \mathrm{TaSe}_{2}\right.$, etc.) [10,18], and topological insulators $\left(\mathrm{Bi}_{2} \mathrm{Se}_{3}, \mathrm{Bi}_{2} \mathrm{Te}_{3}\right.$, etc.) [19,20]. Recently, the deposition of various adsorbates on model topological insulators, such as $\mathrm{Bi}_{2} \mathrm{Se}_{3}$ and $\mathrm{Sb}_{2} \mathrm{Te}_{3}$, has received much consideration [21-39]. Adsorbate deposition provides a route to control the position of the Dirac point relative to the Fermi level [24,29,33,34]. Structural investigations of the impurity-deposited $\mathrm{Bi}_{2} \mathrm{Se}_{3}$ surface reveal partial [23] or almost-complete [25,26] loss of

*http://dipc.ehu.es/gosalvez; miguelangel.gosalvez@ehu.es the adatoms at room and higher temperatures, indicating that the adsorbates may diffuse across the terraces and intercalate at the steps into the van der Waals (vdW) gaps [31]. In addition to general applications in energy storage and synthesis of atom-thick materials, intercalation offers the possibility of adjusting the properties of the host material, e.g., converting a topological insulator into a superconductor [40-46]. In this manner, the understanding of adsorbate diffusion in material-specific energy landscapes remains a prerequisite for clarification of the novel properties observed in new materials.

At the atomistic level, diffusion has a vibrational origin [1-3]. The substrate atoms perform localized oscillations around their equilibrium positions, while the adatoms execute small vibrations about the adsorption sites, occasionally hopping to adjacent sites. The hops occur due to the occasional constructive coupling between the substrate and the adatom vibrations, which provides sufficient energy to jump over the barrier between neighboring sites. This vibrational nature is described by expressing the hop rate of an adparticle as the product of two factors: $v=v_{0} e^{-E_{a} / k_{B} T}$. According to transition state theory (TST) [47-50], the Boltzmann factor $e^{-E_{a} / k_{B} T}$ indicates the probability of performing the jump at temperature $T$ if the energy barrier is $E_{a}$, and the attempt frequency $v_{0}=\frac{k_{B} T}{h} \frac{Z_{T}}{Z_{I}}$ describes how frequently on average the substrate phonons and the adparticle vibrations couple with each other constructively. Here, $k_{B}$ and $h$ are the Boltzmann 

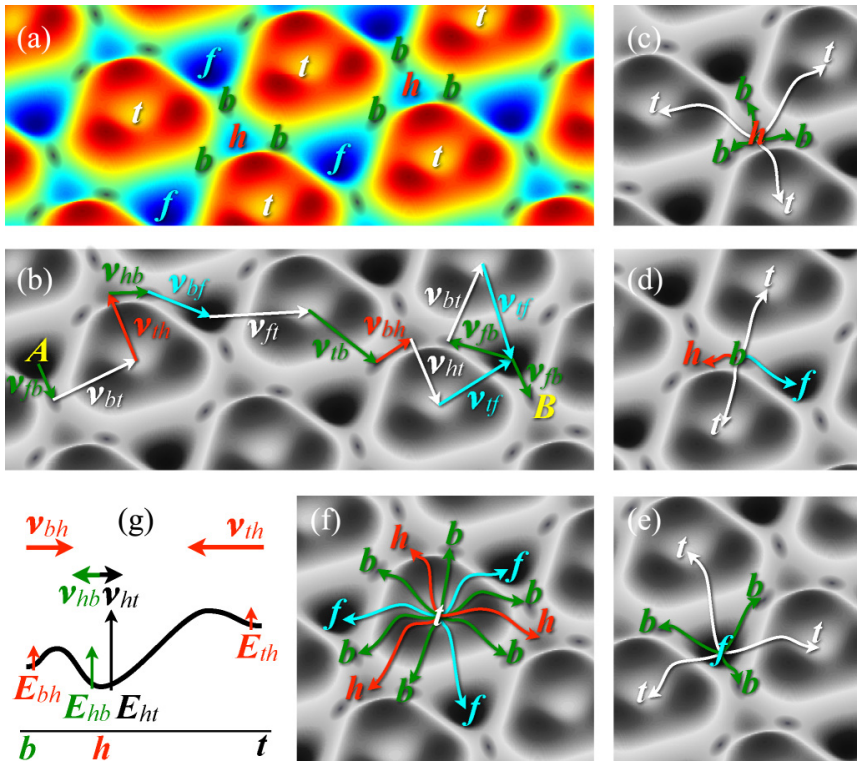

FIG. 1. (a) Example of a complex potential energy landscape for a diffusing particle. Four adsorption site types are indicated: $f, h$, $t$ and $b$. (b) A possible diffusion track (random walk) involving 14 performed jumps at 10 different hop rates $v_{i j}$. (c-f) Possible hops from each site type ( $h, b, f$, and $t$, respectively). Gray-shaded landscapes in (b)-(f) are used to highlight the colored arrows/hops. (g) Energy paths for hops starting/ending in $h$ sites. A longer arrow assigned to a hop rate $\left(v_{t h}, v_{h t}\right.$, etc. $)$ indicates a higher rate.

and Planck constants, respectively, $Z_{T}$ is the partition function for the transition state (or activated complex), calculated for all normal coordinates except the one in which the reaction occurs, and $Z_{I}$ is the partition function for the initial state. In harmonic TST [48,49], the attempt frequency is further simplified to $v_{0}=\left(\Pi_{i=1}^{3 N} v_{i}^{I}\right) /\left(\Pi_{i=1}^{3 N-1} v_{i}^{T}\right)$, i.e., the ratio of the product of the $3 N$ normal mode frequencies in the initial state $\left(v_{i}^{I}\right)$ to the product of the $3 N-1$ normal frequencies in the transition state $\left(v_{i}^{T}\right), N$ being the total number of particles. Generally, TST is valid as long as any trajectory across the transition state does not contain recrossings. This requires that tunneling is absent and the energy barrier is large enough compared to the thermal energy $k_{B} T$. In this study we require that the largest barrier experienced by the adatom satisfies $E_{a}>4 k_{B} T$, where the factor 4 has been taken from Ref. [1]. For complex networks of hops with various energy barriers, the hop rates associated with smaller barriers become so high with increasing temperature that the overall rate is effectively controlled by the lowest rate, i.e., the largest barrier. Thus, we ensure that TST is valid for this barrier.

In this study we are interested in the diffusion of a particle in a complex periodic energy landscape, such as the one shown in Fig. 1(a). One can discern the presence of four adsorption sites (labeled $f, h, t$, and $b$ ), corresponding to the locations where the energy has a local/global minimum. A diffusing particle proceeds by hopping between the sites, as shown schematically in Fig. 1(b). If one focuses on a particular site, as illustrated in Figs. 1(c)-1(f) for $h, b, f$, and $t$, respectively, one may note that each hop requires surpassing a different energy barrier. As further emphasized in Fig. 1(g), the different energy barriers and dissimilar shapes of the energy wells (=dissimilar attempt frequencies) result in different hop rates for the different jumps $\left(v_{h t}, v_{t h}, v_{h b}, v_{b h}\right.$, etc.), including the forward and backward directions. As a result, the random walk between point $A$ and point $B$ in Fig. 1(b) involves as many as 10 hop rates for a total of 14 performed hops. In this study we focus on the analytical description and computational validation of the average distance traveled per unit time by the adparticle, expressing it as a function of the $M$ known hop rates $v_{i j}$ between the $S$ different site types when the number of performed hops $N$ grows very large, i.e., when the diffusion time $t$ becomes arbitrarily long.

The average squared distance covered by a single particle per unit time is a well-defined quantity, known as the tracer diffusion coefficient (or tracer diffusivity) [1],

$$
D_{T}=\frac{1}{2 \alpha} \lim _{t \rightarrow \infty} \frac{\sum_{i=1}^{n}\left\langle\left|\mathbf{r}_{i}(t)-\mathbf{r}_{i}(0)\right|^{2}\right\rangle}{N_{\mathrm{p}} t},
$$

where $\alpha=1,2,3$ is the number of dimensions, $N_{\mathrm{p}}$ is the number of diffusing particles simultaneously present in the system, $\mathbf{r}_{i}(t)$ designates the position of particle $i$ at time $t$, and $\langle\cdot\rangle$ is the ensemble average. Not surprisingly, $D_{T}$ is a function of the number of particles $N_{\mathrm{p}}$ or, equivalently, of the coverage $\theta=N_{\mathrm{p}} / N_{\mathrm{a}}$, where $N_{\mathrm{a}}$ is the number of adsorption sites that may be occupied by the particles. The larger the number of adparticles, the smaller the number of available empty sites that any chosen particle can jump to, thus leading to correlation effects between consecutive hops, also known as memory effects $[1,2]$. This is especially relevant for systems with strong adsorbate-adsorbate interactions [51,52].

We are interested in the low-coverage regime, where the density of diffusing particles is so low that the chance of affecting each other's motion is negligible:

$$
D_{T}^{\theta \approx 0}=\frac{1}{2 \alpha} \lim _{t \rightarrow \infty} \frac{\left\langle|\mathbf{r}(t)-\mathbf{r}(0)|^{2}\right\rangle}{t} .
$$

The low-coverage limit is an important measure, as it provides a simple procedure to compare the typical distances covered by different adsorbates across different substrates [1-5]. Previous analytical work on diluted systems has focused on the determination of the center-of-mass diffusivity for small 2D islands and clusters on metal surfaces based on the master equation [53-55] or the continuous-time random walk formalism $[56,57]$. In the framework of bulk-mediated surface diffusion, Revelli et al. described the average motion of the adsorbed molecules, including both Markovian and non-Markovian desorption, by using the generalized masterequation approach [58]. Condit et al. [59,60] and Birnie [61] derived the overall jump rate for complex, sequential diffusion paths in three-dimensional (3D) crystals, where typical vacancy-interstitial complexes evolve by repeating a particular sequence of hops. Overall, both parts of the present study (see [62] for the second part) generalize this sequential analysis by providing a universal expression for the diffusivity in complex hopping networks where both parallel and sequential diffusion routes are available between the different adsorption sites. The expressions presented in this study for the diffusivity are valid for any combination of values for the hop rates, even if the rates differ by orders of magnitude or become similar/equal. The validity of the proposed expressions is independent of the method used to 
assign values to the hop rates themselves, such as TST or harmonic TST.

Computationally, adsorbate diffusion is traditionally studied by $[1,5]$ (i) first-principles calculations, typically involving the use of density functional theory (DFT) for the determination of the activation barriers and attempt frequencies, which are then passed to other methods; (ii) molecular dynamics simulations, which numerically solve Newton's equations for the substrate and adsorbate atoms based on effective interaction potentials, enabling analysis at the picosecond time scale for systems with $\sim 10^{5}$ atoms; (iii) Langevin models, which describe the adparticle in an effective periodic force field, restricting the analysis to general trends for time scales of picoseconds; and (iv) kinetic Monte Carlo (KMC) simulations, which focus on describing the hops between adjacent basins (rare events) while disregarding all other vibrations, in this way enabling long simulated times (seconds and minutes) with affordable computational resources.

The purpose of this report is to present a general analytical expression for the low-coverage diffusivity in systems that display symmetric hops (Sec. II) and to validate the resulting formulas for illustrative energy landscapes by comparison to KMC simulations (Sec. III), finally applying the formulas to discuss the relative mobility of various adsorbates in the context of topological insulators (Secs. IV and V).

\section{THEORY}

We consider the diffusion of a single particle in a complex, periodic energy landscape, such as the one shown in Fig. 1(a). The diffusing particle may correspond to an adsorbate atom or molecule that moves essentially in two dimensions over a surface. It may also consist of a bulk defect, such as an impurity or vacancy (or a pair/cluster of these) traveling in three dimensions within a crystal. While the energy landscape corresponds to an energy surface for the 2D case, for bulk diffusion it corresponds to a 3D scalar field, characterized by finite volumes where the energy increases alternating with volumes where it decreases. Although more difficult to represent graphically, the periodicity of the defect energy as it diffuses within the material allows describing the 3D case by the same formalism as in the $2 \mathrm{D}$ case. Thus, although the graphical illustrations presented below are geared for two dimensions, the underlying mathematical derivations and main result are valid in three dimensions as well.

\section{A. Definitions}

Let us consider a system with $S$ different site types, such as the one shown in Figs. 2(a) and 2(b) for $S=4$ or Fig. 2(c) for $S=3$. For a generic hop from site type $i$ to site type $j$ $(i, j=1, \ldots, S)$ the hop distance $l_{i j}$, hop rate $v_{i j}$, and hop multiplicity $m_{i j}$ are assumed to be known. $m_{i j}$ is the number of equivalent hops from the initial site type $i$ to nearby end sites of type $j$. In this paper we focus on systems where $m_{i j} \geqslant 2$ and the corresponding end sites are symmetrically located with respect to the initial site. In this manner, the center of mass of the end sites coincides with the location of the initial site, and the hops are termed symmetric. In two dimensions, the directions along which such symmetric hops can occur (a) Triangular

(b) Rectangular

(c) Square
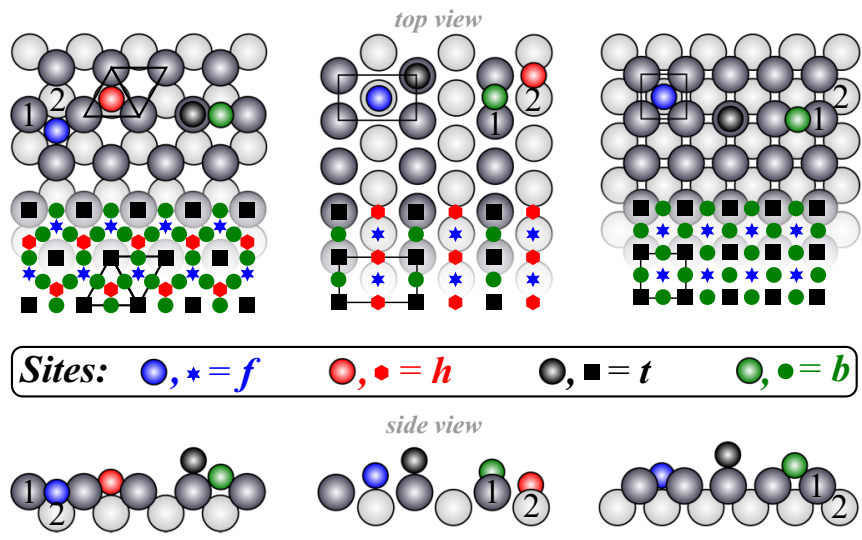

$0, \bullet=h$

$0, \square=t$

$0, \bullet=b$

side view
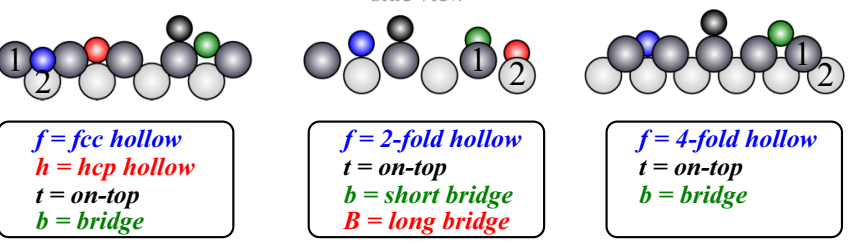

FIG. 2. Schematic of three typical surfaces with (a) triangular symmetry [e.g., fcc(111) and hcp(0001)], (b) rectangular symmetry [e.g., fcc(110)], and (c) square symmetry [e.g., fcc(100)]. We refer to typical adsorption sites as $f$ (for fcc hollow), $h$ (for hcp hollow), $t$ (for on top), $b$ (for bridge/short bridge), and $B$ (for long bridge).

are necessarily related by rotational ( $n$-fold) symmetry: two opposite directions for $n=2$ and the trigonal-planar, squareplanar, and hexagonal-planar geometries for $n=3,4$, and 6 , respectively. In three dimensions, the directions of such symmetric hops correspond to the tetrahedral, octahedral, cubic, and cuboctahedral geometries $(n=4,6,8$, and 12, respectively). The next part of the present study [62] provides a detailed analysis of the diffusivity in systems containing asymmetric hops that do not satisfy this definition.

We consider a new variable, the rateplicity $\mu_{i j}$, defined as the product of the hop rate and the multiplicity:

$$
\mu_{i j}=m_{i j} v_{i j}
$$

For an atom located at site $i$, we also define the escape rate $R_{i}$ as the sum of the rateplicities to all accessible neighboring sites,

$$
R_{i}=\Sigma_{k} \mu_{i k},
$$

and the escape time $\tau_{i}$ as the inverse of the escape rate,

$$
\tau_{i}=\frac{1}{R_{i}} .
$$

Here, the summation runs through all site types, i.e., $\Sigma_{k}=$ $\Sigma_{k=1}^{S}$. From one hop to the next, time advances by an amount equal to the inverse of the total rate. For a system with a single adatom, the total rate is equal to the current value of the escape rate. Thus, the time increments for different hops from the same site type $i$ to different, accessible site types $j$ and $j^{\prime}$ are equal:

$$
\tau_{i j}=\tau_{i j^{\prime}}=\tau_{i}, \quad j \neq j^{\prime} .
$$

In addition, we define the escape rate to all other sites $R_{i}^{\neq}$as the sum of the rateplicities to all accessible neighboring sites 
that are different from the origin $i$ of the hop:

$$
R_{i}^{\neq}=\Sigma_{k \neq i} \mu_{i k}=R_{i}-\mu_{i i} .
$$

We also define the hop probability $\pi_{i j}$ from site $i$ to site $j$ as the ratio of the rateplicity $\mu_{i j}$ to the escape rate $R_{i}$ :

$$
\pi_{i j}=\frac{\mu_{i j}}{R_{i}}
$$

$\pi_{i j}$ describes the relative rate of hopping from site $i$ to site $j$ in comparison to the total rate of hopping from $i$ to any accessible neighboring site. As the normalized rateplicity, $\pi_{i j}$ satisfies the following three important relations:

$$
\begin{gathered}
\Sigma_{j} \pi_{i j}=1, \\
\pi_{i j^{\prime}}=\frac{\mu_{i j^{\prime}}}{\mu_{i j}} \pi_{i j}, \\
\pi_{i j}=\mu_{i j} \tau_{i j} .
\end{gathered}
$$

This is demonstrated by directly using the definition [Eq. (8)] in Eqs. (9)-(11). Similarly, we define the conditional hop probability from site $i$ to site $j\left(p_{i j}\right)$ as the product of the equilibrium probability that the atom is located at site $i\left(p_{i}\right)$ and the hop probability from site $i$ to site $j\left(\pi_{i j}\right)$ :

$$
p_{i j}=p_{i} \pi_{i j} .
$$

Making use of the general principle of detailed balance, the conditional probability of performing one process must be equal to the conditional probability of performing the reverse process $\left(p_{i j}=p_{j i}\right)$ :

$$
p_{i} \pi_{i j}=p_{j} \pi_{j i}
$$

Summing over $j$ in Eq. (13) we have $(i=1,2, \ldots, S)$ :

$$
\begin{gathered}
\Sigma_{j} p_{i j}=\Sigma_{j} p_{j i}, \\
\Sigma_{j} p_{i} \pi_{i j}=\Sigma_{j} p_{j} \pi_{j i}, \\
p_{i}=\Sigma_{j} p_{j} \pi_{j i}, \\
p_{i}=\Sigma_{k} p_{k i} .
\end{gathered}
$$

This means that the probability of escaping from site type $i$ [left-hand side of Eq. (14)] is equal to the probability of arriving at that site type $i$ [right-hand side of Eq. (14)]. Based on Eq. (17), this also means that $p_{i}$ can be regarded as the probability of arriving at site $i$ from any other hop-connected neighboring site. Using Eq. (8) in Eq. (12) we can also rewrite:

$$
p_{i}=R_{i} \frac{p_{i j}}{\mu_{i j}} .
$$

Finally, combining Eqs. (12) and (17) one obtains

$$
\begin{aligned}
\pi_{i j} & =\frac{p_{i j}}{\Sigma_{k} p_{k i}}, \\
\frac{p_{i j}}{\pi_{i j}} & =\Sigma_{k} p_{k i},
\end{aligned}
$$

and rewriting Eq. (20) for two different hops from the same site gives

$$
\begin{aligned}
& p_{i j^{\prime}}=\frac{\pi_{i j^{\prime}}}{\pi_{i j}} p_{i j}, \\
& p_{i j^{\prime}}=\frac{\mu_{i j^{\prime}}}{\mu_{i j}} p_{i j},
\end{aligned}
$$

where Eq. (10) has been used to go from Eq. (21) to (22).

\section{B. Analytical solution}

The conditional probabilities play a key role in our description of the low-coverage tracer diffusivity. The starting point is to express $D_{T}^{\theta \approx 0}$ [Eq. (2)] as the ratio of the average squared hop distance to the average time increment, formulating the two averages as weighted sums of the corresponding variables (squared hop distance and escape time) with the conditional probabilities as weights:

$$
D_{T}^{\theta \approx 0}=\frac{1}{2 \alpha} \underbrace{\overbrace{\sum_{i, j} p_{i j} l_{i j}^{2}}^{\sum_{i, j} p_{i j} \tau_{i j}}}_{Q} .
$$

The goal is to rewrite Eq. (23) explicitly in terms of the known hop rates and multiplicities or, equivalently, as a function of the rateplicities.

We start by using Eq. (22) to express all conditional probabilities that share the same hop origin $(i)$ in terms of the corresponding self-hop (ii):

$$
p_{i j}=\mu_{i j} \frac{p_{i i}}{\mu_{i i}} \quad(i, j=1,2, \ldots, S) .
$$

Accordingly, from Eq. (23) we have

$$
\begin{gathered}
P=\Sigma_{i, j} p_{i j} l_{i j}^{2}=\Sigma_{i, j} \frac{p_{i i}}{\mu_{i i}} \mu_{i j} l_{i j}^{2}=\Sigma_{i} \frac{p_{i i}}{\mu_{i i}}\left(\Sigma_{j} \mu_{i j} l_{i j}^{2}\right), \\
Q=\Sigma_{i, j} p_{i j} \tau_{i j}=\Sigma_{i, j} \frac{p_{i j}}{\mu_{i j}} \pi_{i j}=\Sigma_{i, j} \frac{p_{i i}}{\mu_{i i}} \pi_{i j} \\
=\Sigma_{i} \frac{p_{i i}}{\mu_{i i}}\left(\Sigma_{j} \pi_{i j}\right)=\Sigma_{i} \frac{p_{i i}}{\mu_{i i}}
\end{gathered}
$$

where we have used Eq. (11) to substitute $\tau_{i j}=\pi_{i j} / \mu_{i j}$ in Eq. (26), and Eq. (9) to substitute $\Sigma_{j} \pi_{i j}=1$ in Eq. (27). Thus, Eq. (23) becomes

$$
\begin{aligned}
D_{T}^{\theta \approx 0} & =\frac{1}{2 \alpha} \frac{P}{Q}=\frac{1}{2 \alpha} \frac{\Sigma_{i} \frac{p_{i i}}{\mu_{i i}}\left(\Sigma_{j} \mu_{i j} l_{i j}^{2}\right)}{\Sigma_{i} \frac{p_{i i}}{\mu_{i i}}} \\
& =\frac{1}{2 \alpha} \Sigma_{i} w_{i}\left(\Sigma_{j} \mu_{i j} l_{i j}^{2}\right) .
\end{aligned}
$$

Note that in Eq. (29) we have defined the coefficients,

$$
w_{i}=\underbrace{\frac{\frac{p_{i i}}{\mu_{i i}}}{\Sigma_{j} \frac{p_{j j}}{\mu_{j j}}}}_{T}=\underbrace{\frac{\frac{p_{i k}}{\mu_{i k}}}{\Sigma_{j} \frac{p_{j k}}{\mu_{j k}}}}_{U}=\underbrace{\frac{\frac{p_{i}}{R_{i}}}{\Sigma_{j} \frac{p_{j}}{R_{j}}}}_{V}=\underbrace{\frac{p_{i} \tau_{i}}{\Sigma_{j} p_{j} \tau_{j}}}_{W},
$$

where Eq. (24) has been used in the transformation $T \rightarrow U$, Eq. (18) in $U \rightarrow V$, and Eq. (5) in $V \rightarrow W$. According to $W$, the coefficient $w_{i}$ is the fraction of time spent at site type $i$ relative to the time spent at all site types. In other words, $w_{i}$ is the probability of residence at site $i$ or the occupancy 
probability of site type $i$. Thus, we refer to $w_{i}$ simply as the occupancy of site $i$. Note that the sum of all occupancies is 1 $\left(\Sigma_{i} w_{i}=1\right)$.

The occupancy, $w_{i}$, should not be confused with the equilibrium probability, $p_{i}$. According to $V$ in Eq. (30), the equilibrium probability of finding the adatom at site type $i$ is

$$
p_{i}=\frac{w_{i} R_{i}}{\Sigma_{j} w_{j} R_{j}} .
$$

Using this relation, Eq. (13) for detailed balance becomes

$$
w_{i} \mu_{i j}=w_{j} \mu_{j i},
$$

and summing over $j$ in Eq. (32) gives $(i=1,2, \ldots, S)$

$$
w_{i} \Sigma_{j} \mu_{i j}=\Sigma_{j} w_{j} \mu_{j i} \text {. }
$$

Equation (33) is very important. The left-hand side can be understood as the flux (of particles) out of site $i$, and the right-hand side as the flux into site $i$. Thus, the net flux is 0 as a consequence of detailed balance. In addition, Eq. (33) provides a system of equations for the occupancies, $w_{i}$, which are needed to determine the diffusivity [Eq. (29)]. Solving this system is considered next.

Using Eq. (4) into Eq. (33) and the definition in Eq. (7), one gets $(i=1,2, \ldots, S)$ :

$$
\begin{gathered}
w_{i} R_{i}-\Sigma_{j} w_{j} \mu_{j i}=0, \\
w_{i}\left(R_{i}-\mu_{i i}\right)-\Sigma_{j \neq i} w_{j} \mu_{j i}=0, \\
w_{i} R_{i}^{\neq}-\Sigma_{j \neq i} w_{j} \mu_{j i}=0 .
\end{gathered}
$$

Equation (36) is especially useful. One of the $S$ equations contained in it is redundant due to the fact that the sum of all occupancies is $1\left(\Sigma_{i} w_{i}=1\right)$. Taking the equation for $i=1$ to be the redundant one and expressing Eq. (36) in matrix form leads to

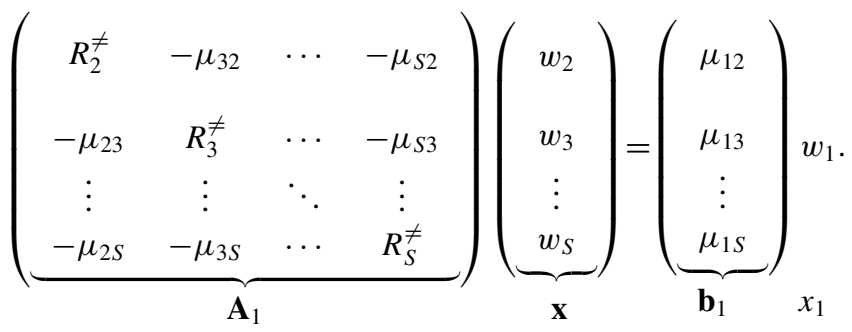

Thus, we need to solve the matrix equation $\mathbf{A}_{1} \cdot \mathbf{x}=\mathbf{b}_{1} x_{1}$ for vector $\mathbf{x}$, with components $x_{i}=w_{i}$, where $i=2, \ldots, S$. Using Cramer's rule we have $x_{i}=x_{1} \operatorname{det}\left(\mathbf{A}_{i}\right) / \operatorname{det}\left(\mathbf{A}_{1}\right)$, where $\mathbf{A}_{i}$ is the matrix that results from substitution of column $i-1$ in matrix $\mathbf{A}_{1}$ by vector $\mathbf{b}_{1}$ :

$$
\mathbf{A}_{i} \equiv \mathbf{A}_{1}\left(\left[\mathbf{b}_{1}\right]_{i-1}\right) \quad(i=2, \ldots, S) .
$$

Thus, the solution of Eq. (37) is

$$
w_{i}=\frac{B_{i}}{B_{1}} w_{1} \quad(i=2, \ldots, S)
$$

where

$$
B_{i}=\operatorname{det}\left(\mathbf{A}_{i}\right) \quad(i=1, \ldots, S) .
$$

Finally, taking into account that $\Sigma_{i} w_{i}=1$ we have

$$
w_{i}=\frac{B_{i}}{\Sigma_{j} B_{j}} \quad(i=2, \ldots, S) .
$$

In practice, Eq. (41) is useful to determine the coefficients $w_{i}$ that appear in Eq. (29). We stress that Eq. (36) and its solution, Eq. (41), are valid for any value of $S$.

Equation (29) is the central result of the present paper (for systems with symmetric hops only). Valid for any number of different site types $S$, it means that the total diffusivity can be written as a weighted sum of partial diffusivities $\left(\Sigma_{j} \mu_{i j} l_{i j}^{2}\right)$, which contain the rateplicities and hop lengths for all jumps from every site type $i$ to any accessible site type $j$. To calculate the overall diffusivity we need to determine the occupancy $w_{i}=B_{i} / \Sigma_{j} B_{j}$ for each site type $i$ that acts as a hop origin, multiplying it by the sum of several $\mu_{i j} l_{i j}^{2}$ terms, one for each different site $j$ accessed from $i$ and, finally, summing over all considered hop origins $i$. In order to determine each $B_{i}$ we can build matrix $\mathbf{A}_{i}$ in different ways (see Sec. II C) and apply the definition $B_{i}=\operatorname{det}\left(\mathbf{A}_{i}\right)$. Alternatively, the occupancies $w_{i}$ can be determined directly from the rateplicities using Eq. (51) (see Sec. II C).

\section{Ways to determine $B_{i}$ and $w_{i}$}

Due to the redundancy of one of the relations in Eq. (36), every determinant $B_{i}=\operatorname{det}\left(\mathbf{A}_{i}\right)$ can be calculated using different forms for matrix $\mathbf{A}_{i}$, all of which will generate the same value. Focusing on $\mathbf{A}_{2}$ for instance, if the equation for site type $i=1$ is chosen as the redundant one, then $\mathbf{A}_{1}$ and $\mathbf{b}_{1}$ are given by Eq. (37), and $\mathbf{A}_{2}$ is

$$
\mathbf{A}_{2} \quad \equiv \mathbf{A}_{1}\left(\left[\mathbf{b}_{1}\right]_{1}\right)=\left(\begin{array}{cccc}
\mu_{12} & -\mu_{32} & \cdots & -\mu_{S 2} \\
\mu_{13} & R_{3}^{\neq} & \cdots & -\mu_{S 3} \\
\vdots & \vdots & \ddots & \vdots \\
\mu_{1 S} & -\mu_{3 S} & \cdots & R_{S}^{\neq}
\end{array}\right) .
$$

However, we can alternatively choose the equation for $i=2$ to be the redundant one, in which case we directly have an expression for $\mathbf{A}_{2}$ :

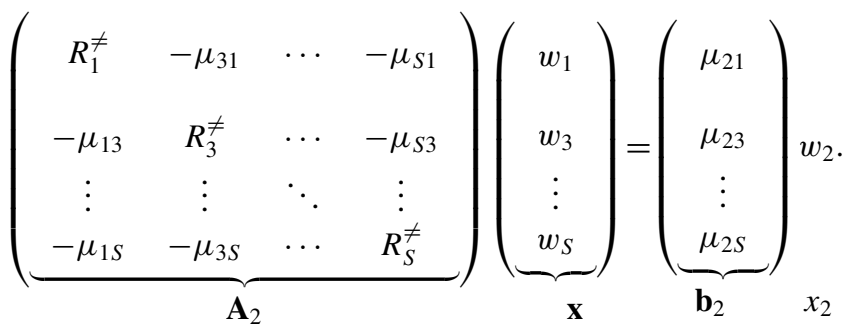

Similarly, we can choose the redundant equation for any other site type and express $\mathbf{A}_{2}$ accordingly. By writing out the determinants of the different forms for $\mathbf{A}_{2}$ it can be verified that they all give the same expression for $B_{2}$. Due to this redundancy, the particular form of other $\mathbf{A}_{i}$ 's can also be chosen rather freely in practice.

Typically some/many elements of the $\mathbf{A}_{i}$ matrices are 0 and the determinants result in relatively simple expressions 
for the $B_{i}$ coefficients and $w_{i}$ occupancies. In particular, when the number of different adsorption sites $S$ is small enough (e.g., $S=2,3$ ) we can explicitly write the following, simple expressions:

$$
\begin{gathered}
B_{i}=\mu_{j i} \quad(S=2 ; i, j=1,2 ; i \neq j), \\
B_{i}=\mu_{j k} \mu_{k i}+\mu_{k j} \mu_{j i}+\mu_{j i} \mu_{k i} \\
(S=3 ; i, j, k=1,2,3 ; i \neq j \neq k) .
\end{gathered}
$$

As an alternative, we may express Eq. (39) as follows:

$$
\frac{B_{i}}{B_{j}}=\frac{w_{i}}{w_{j}}=\frac{\mu_{j i}}{\mu_{i j}} \quad(i, j=1, \ldots, S),
$$

where Eq. (32) (detailed balance) has been used. This provides another formulation to obtain the $w_{i}$ occupancies:

$$
\begin{aligned}
w_{1} & =\frac{B_{1}}{B_{1}+B_{2}+\cdots+B_{S}} \\
& =\frac{1}{1+\frac{B_{2}}{B_{1}}+\cdots+\frac{B_{S}}{B_{1}}}=\frac{1}{1+\frac{\mu_{12}}{\mu_{21}}+\cdots+\frac{\mu_{1 S}}{\mu_{S 1}}} \\
& =\frac{\frac{B_{1}}{B_{2}}}{\frac{B_{1}}{B_{2}}+1+\cdots+\frac{B_{S}}{B_{2}}}=\frac{\frac{\mu_{21}}{\mu_{12}}}{\frac{\mu_{21}}{\mu_{12}}+1+\cdots+\frac{\mu_{2 S}}{\mu_{S 2}}} \\
& =\cdots \\
& =\frac{\frac{B_{S 1}}{B_{S}}}{\frac{B_{1}}{B_{S}}+\frac{B_{2}}{B_{S}}+\cdots+1}=\frac{\mu_{S 1}}{\frac{\mu_{S 1}}{\mu_{1 S}}+\frac{\mu_{S 2}}{\mu_{2 S}}+\cdots+1},
\end{aligned}
$$

with similar equations for $w_{2}, \ldots, w_{S}$. This can be summarized by the following, single expression:

$$
w_{i}=\underbrace{\frac{B_{i}}{\Sigma_{k} B_{k}}}=\underbrace{\frac{\frac{B_{i}}{B_{j}}}{\Sigma_{k} \frac{B_{k}}{B_{j}}}}=\underbrace{\frac{\frac{\mu_{j i}}{\mu_{i j}}}{\Sigma_{k} \frac{\mu_{j k}}{\mu_{k j}}}}
$$$$
\text { (a) } \quad(b) \quad(c)
$$

$$
(i, j=1, \ldots, S) \text {, }
$$

where any $j$ can be used in (51b) and (51c). For simplicity, however, we typically use the same $j$ for all $i$ 's.

Equation (51c) usually provides the shortest route to obtain the occupancies. It explicitly contains the rateplicities, and thus, there is no need to build the $\mathbf{A}_{i}$ matrices. One must be careful, however, since Eq. (51) may involve divisions by 0 when $S \geqslant 3$. The case $S=2$ is special, since the diffusing particle can hop only from one site type (say 1) to the other (say 2 ), and vice versa. Thus, the hop rates between the two different sites are different from 0 . Note that the hop rates between identical sites do not appear in Eq. (51) [since they lead to ones] and, thus, are irrelevant for determination of the occupancies $w_{i}$. For $S \geqslant 3$, however, some hops between different sites may be forbidden, in which case the corresponding hop rates will be effectively 0 . Thus, Eq. (51) may contain indeterminate values of type $\frac{\mu_{m n}}{\mu_{n m}}=\frac{0}{0}$. We deal with this next.

\section{Forward/backward fundamental paths}

To handle the indeterminate values, we make use of certain relations between the rateplicities that appear on specific sequences of hops, here referred to as fundamental paths. A fundamental path is a sequence of hops that starts and ends at the same site type and visits other site types at most once (see Fig. 3). Every fundamental path has an associated opposite path, in which the sequence of hops is exactly the opposite. As an example, the fundamental paths 1231 and 1321 start and end at site 1 , but they visit sites 2 and 3 in opposite order. In this study, the two opposite paths are referred to as the forward path (FW) and backward path (BW). As shown below, a necessary condition for detailed balance to be fulfilled is that the product of the rateplicities must be equal along the forward and backward paths.

The announced relations between the rateplicity products can be obtained by directly comparing the right-hand sides of Eqs. (48)-(50). For $S=3$, equating the right-hand side of Eqs. (48) and (49) [or (48) and (50), or (49) and (50)] gives

$$
\mu_{12} \mu_{23} \mu_{31}=\mu_{13} \mu_{32} \mu_{21} .
$$

Thus, in this case the product of the rateplicities appearing in the forward path $(\mathrm{FW}=1231)$ must be equal to that of the rateplicities in the backward path $(\mathrm{BW}=1321)$. Note that Eq. (52) also applies to the paths starting and ending at site 2 $\left(\mu_{23} \mu_{31} \mu_{12}=\mu_{21} \mu_{13} \mu_{32}\right.$ for $\mathrm{FW}=2312$ and $\left.\mathrm{BW}=2132\right)$ and site $3\left(\mu_{31} \mu_{12} \mu_{23}=\mu_{32} \mu_{21} \mu_{13}\right.$ for $\mathrm{FW}=3123$ and $\mathrm{BW}=3213$ ).

For $S=4$, equating the right-hand side of Eqs. (48)-(50) leads to

$$
\begin{aligned}
\mu_{12} \mu_{23} \mu_{31} & =\mu_{13} \mu_{32} \mu_{21}, \\
\mu_{12} \mu_{24} \mu_{41} & =\mu_{14} \mu_{42} \mu_{21}, \\
\mu_{13} \mu_{34} \mu_{41} & =\mu_{14} \mu_{43} \mu_{31}, \\
\mu_{12} \mu_{23} \mu_{34} \mu_{41} & =\mu_{14} \mu_{43} \mu_{32} \mu_{21}, \\
\mu_{12} \mu_{24} \mu_{43} \mu_{31} & =\mu_{13} \mu_{34} \mu_{42} \mu_{21}, \\
\mu_{13} \mu_{32} \mu_{24} \mu_{41} & =\mu_{14} \mu_{42} \mu_{23} \mu_{31}
\end{aligned}
$$

for fundamental paths involving three and four hops, respectively. Similarly, for larger values of $S$, additional products are obtained for all fundamental paths involving three hops, four hops, and so on, up to $S$ hops.

Recalling that $\mu_{i j}=m_{i j} v_{i j}$, where $m_{i j}$ is the multiplicity and $v_{i j}$ the hop rate, Eq. (53) reads

$$
m_{12} m_{23} m_{31} v_{12} v_{23} v_{31}=m_{13} m_{32} m_{21} v_{13} v_{32} v_{21} .
$$

Since $m_{i j}$ is the number of equivalent hops from site $i$ to site $j$, the product $m_{12} m_{23} m_{31}$ appearing on the left-hand side of Eq. (59) represents the total number of equivalent paths of type $\mathrm{FW}=1231$. This is visually shown in Fig. 3(a). Similarly, the product $m_{13} m_{32} m_{21}$ appearing on the right-hand side of Eq. (59) represents the total number of equivalent paths for $\mathrm{BW}=1321$ (opposite order). Since the actual geometry is the same, the total number of equivalent paths is necessarily the same for both the forward and the backward paths. In other words, $m_{12} m_{23} m_{31}=m_{13} m_{32} m_{21}$. Similarly, when substituting $\mu_{i j}=m_{i j} v_{i j}$ in Eqs. (54)-(58), Figs. 3(b)-3(f) show that the resulting products of the multiplicities are always 


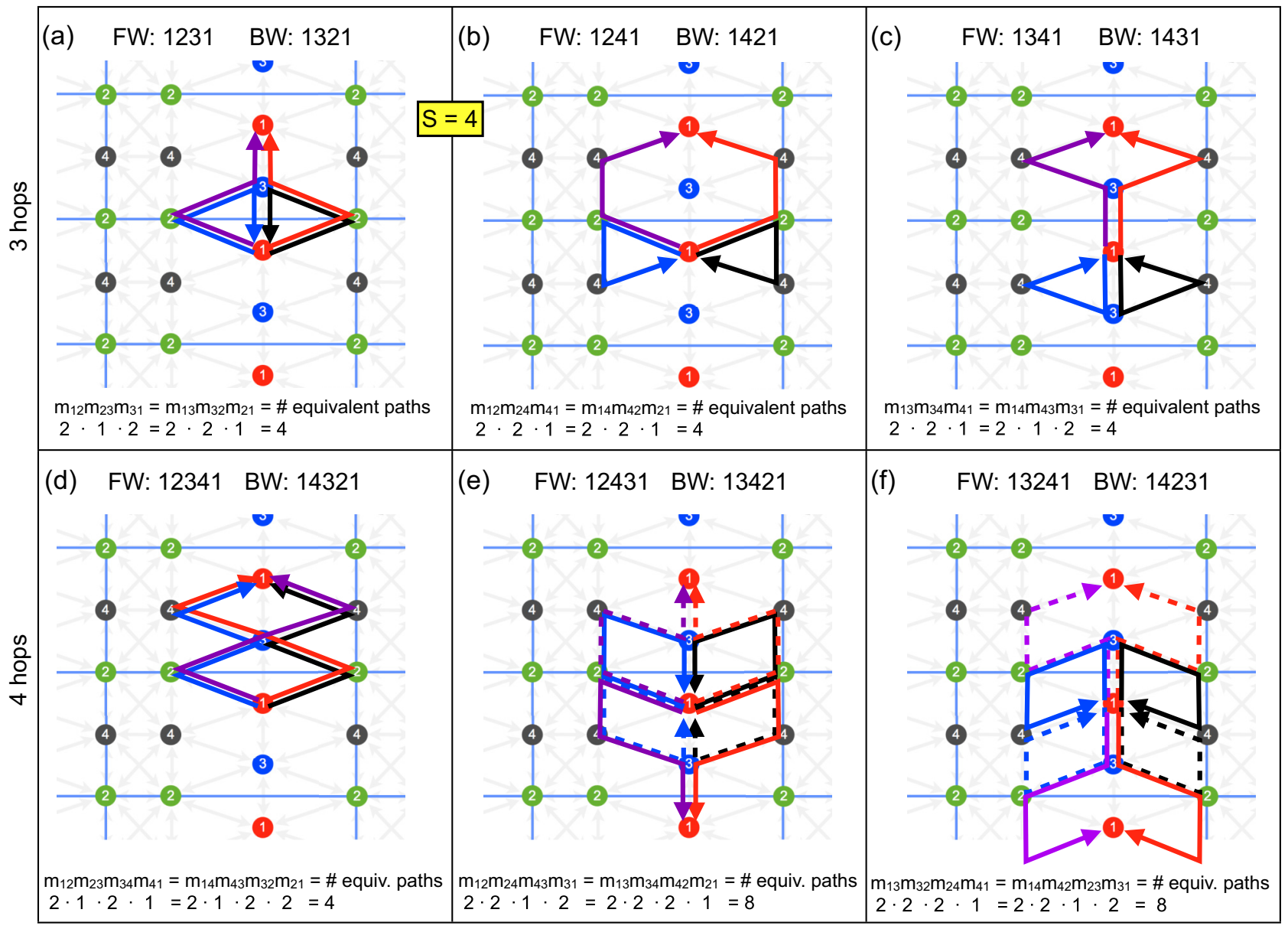

(g)

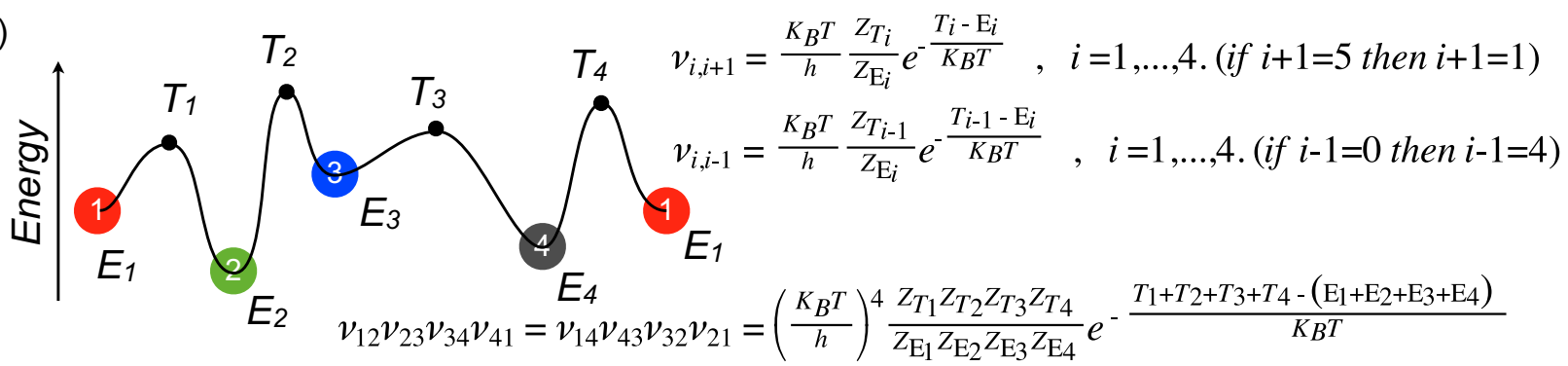

FIG. 3. Fundamental paths for a system with four site types. FW, forward path; BW, backward path. (a-d) All four equivalent paths for $\mathrm{FW}=1231,1241,1341$, and 12341, respectively, and $\mathrm{BW}=1321,1421,1431$, and 14321, respectively. (e, f) All eight equivalent paths for $\mathrm{FW}=12431$ and 13241 , respectively, and $\mathrm{BW}=13421$ and 14231 , respectively. $(\mathrm{g})$ Demonstration of the equality $v_{12} v_{23} \ldots v_{S 1}=$ $v_{1 S} v_{S S-1} \ldots v_{21}$ for $S=4$.

equal for the forward and backward paths. In this manner, the multiplicities cancel out and Eqs. (53)-(58) are simplified to

$$
\begin{aligned}
& v_{12} \nu_{23} \nu_{31}=v_{13} \nu_{32} \nu_{21} \text {, } \\
& v_{12} v_{24} v_{41}=v_{14} v_{42} v_{21} \text {, } \\
& v_{13} v_{34} v_{41}=v_{14} v_{43} v_{31}, \\
& \nu_{12} \nu_{23} \nu_{34} v_{41}=v_{14} v_{43} \nu_{32} \nu_{21} \text {, } \\
& v_{12} v_{24} v_{43} \nu_{31}=v_{13} v_{34} v_{42} v_{21} \text {, } \\
& \nu_{13} \nu_{32} \nu_{24} \nu_{41}=v_{14} \nu_{42} \nu_{23} \nu_{31}
\end{aligned}
$$

Equations (60)-(65) for $S=4$ (and similar equations for other values of $S$ ) are always satisfied, provided that the hop rates $v_{i j}$ are described using Boltzmann factors, as shown in Fig. 3(g) Note that, within TST, typically the Boltzmann prefactors will be different for different hop types starting at the same site. We conclude that, along any fundamental path, the forward and backward products of the hop rates are equal.

This provides a procedure to handle the indeterminate values of type $\frac{\mu_{i j}}{\mu_{j i}}=\frac{0}{0}$ in Eq. (51). In fact, we may consider any pair of forward/backward fundamental paths that contains $\mu_{i j}$ and $\mu_{j i}$ and rewrite their ratio as

$$
\begin{gathered}
\frac{\mu_{i j}}{\mu_{j i}}=\frac{\mu_{i k} \mu_{k j}}{\mu_{j k} \mu_{k i}} \quad \text { (for } 3 \text { hops, } i \neq j \neq k \text { ), } \\
\frac{\mu_{i j}}{\mu_{j i}}=\frac{\mu_{i k} \mu_{k l} \mu_{l j}}{\mu_{j l} \mu_{l k} \mu_{k i}} \quad \text { (for } 4 \text { hops, } i \neq j \neq k \neq l \text { ), }
\end{gathered}
$$


TABLE I. Examples of low-coverage tracer diffusivities $\left(D_{T}^{\theta \approx 0}\right)$ for different combinations of hop rates between standard adsorption sites on square, rectangular, and triangular lattices. Site labels: $f$-fourfold hollow (square)/twofold hollow (rectangular)/fcc hollow (triangular); $h$-hcp hollow (triangular); $t$-on top; $b$-bridge (square)/short bridge (rectangular); $B$-long bridge (rectangular). Additional expressions for other hop combinations are listed in Tables III and IV. Expressions $[a],[b],[c],[d],[e]$, and $[f]$ are all equivalent since $v_{t f} v_{f b} v_{b t}=v_{t b} v_{b f} v_{f t}$ (see Sec. II D). Similarly, $[g]$ and $[h]$ are equal since $v_{t B} v_{B f} v_{f b} v_{b t}=v_{t b} v_{b f} v_{f B} v_{B t}$. Also, $[i]$ and $[j]$ are equal since $v_{t f} v_{f h} v_{h t}=v_{t h} v_{h f} v_{f t}$. There are other equivalent forms for $[g]-[j]$ (see text). Sym, symmetry.

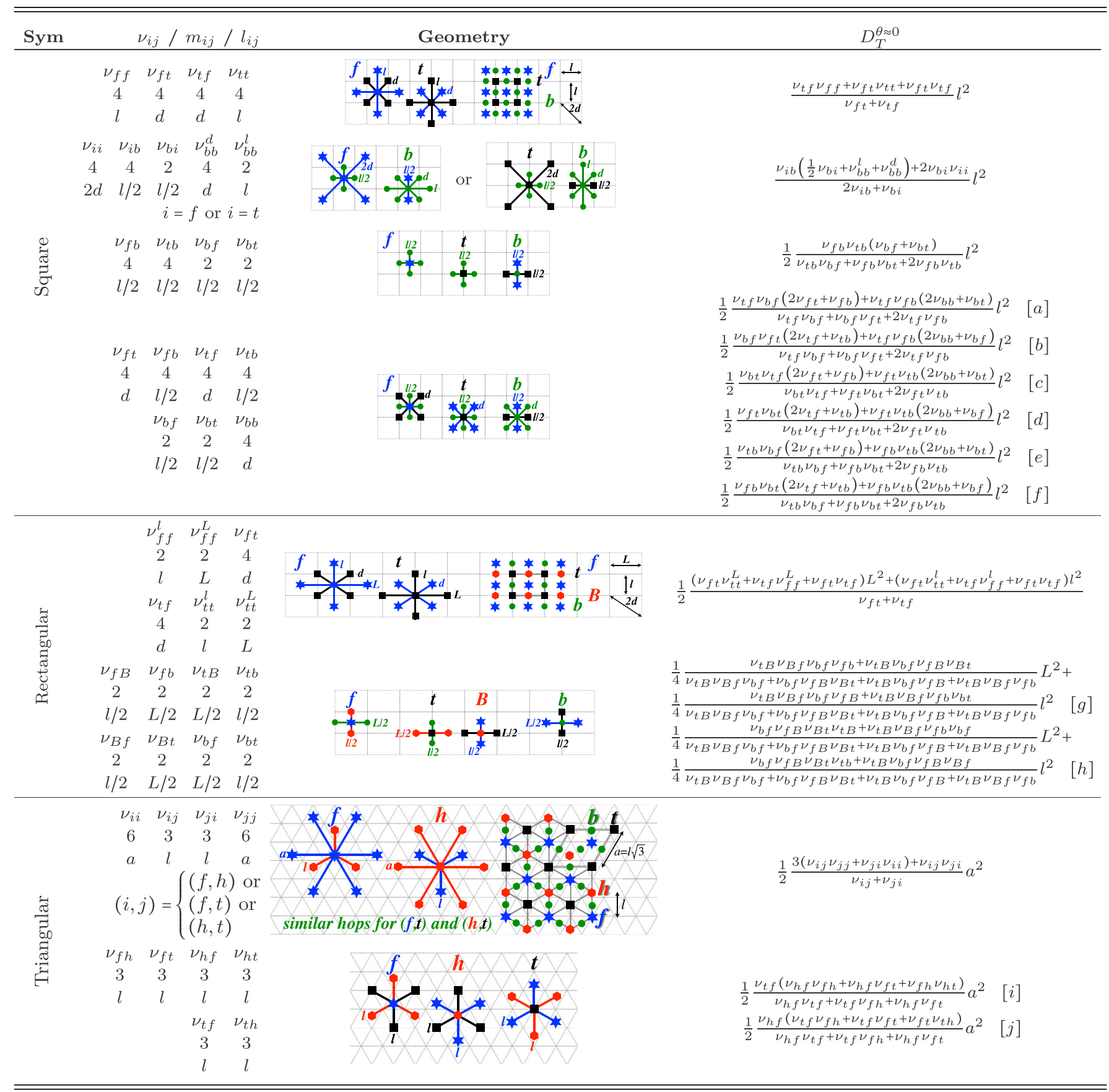

and similar relations for fundamental paths with larger numbers of hops.

As an example, consider the system shown in the third row of Table I, where the hops $v_{f b}, v_{t b}, v_{b f}$, and $v_{b t}$ are allowed and have multiplicities $4,4,2$, and 2 , respectively, all involving a hop distance of $l / 2$. However, the hops $v_{f t}, v_{t f}$ are forbidden, i.e., $v_{f t}=v_{t f}=0$. Applying Eq. (51) leads to

$$
w_{f}=\frac{B_{f}}{B_{f}+B_{t}+B_{b}}=\frac{1}{1+\frac{B_{t}}{B_{f}}+\frac{B_{b}}{B_{f}}}=\frac{1}{1+\frac{\mu_{f t}}{\mu_{t f}}+\frac{\mu_{f b}}{\mu_{b f}}} .
$$


Here, $\frac{\mu_{f b}}{\mu_{b f}}=2 \frac{v_{f b}}{v_{b f}}$ but $\frac{\mu_{f t}}{\mu_{t f}}=\frac{0}{0}$ is indeterminate. To resolve the latter, we use the fact that $\mu_{f t} \mu_{t b} \mu_{b f}=\mu_{f b} \mu_{b t} \mu_{t f}$. In other words,

$$
\frac{\mu_{f t}}{\mu_{t f}}=\frac{\mu_{f b} \mu_{b t}}{\mu_{t b} \mu_{b f}}=\frac{v_{f b} v_{b t}}{v_{t b} v_{b f}} .
$$

Thus, we obtain

$$
w_{f}=\frac{1}{1+\frac{v_{f b} v_{b t}}{v_{t b} v_{b f}}+2 \frac{v_{f b}}{v_{b f}}}=\frac{v_{t b} v_{b f}}{v_{t b} v_{b f}+v_{f b} v_{b t}+2 v_{f b} v_{t b}}
$$

Similarly,

$$
\begin{aligned}
& w_{t}=\frac{v_{f b} v_{b t}}{v_{t b} v_{b f}+v_{f b} v_{b t}+2 v_{f b} v_{t b}}, \\
& w_{b}=\frac{2 v_{f b} v_{t b}}{v_{t b} v_{b f}+v_{f b} v_{b t}+2 v_{f b} v_{t b}} .
\end{aligned}
$$

\section{E. Obtaining formulas for the diffusivity}

Table I lists a few example formulas obtained using Eq. (29) by applying Eq. (51) to obtain the occupancies and Eqs. (66) and (67) to resolve any indeterminate values $\frac{\mu_{i j}}{\mu_{j i}}=\frac{0}{0}$, if needed. Equivalently, one may use Eqs. (40) and (41) to obtain the occupancies $w_{i}$. The table lists results for various systems, including square, rectangular, and triangular lattices. Additional expressions for other hop combinations in the rectangular and triangular lattices are provided in Tables III and IV.

When $S>2$, the diffusivity typically can be described by several equivalent expressions, as shown by formulas $[a]-[f]$ in row 4 of Table I. This is due to the fact that the occupancies appearing in Eq. (29) can be described by mutually equivalent expressions [see Eqs. (48)-(50)] and also the products of the rates can be written out in various equivalent forms, according to the underlying fundamental paths [see Eqs. (60)-(65)]. Since expressions $[a]$ through $[f]$ are equal, the diffusivity can also be described as a combination of those expressions. In fact, when applying Eq. (29) one directly obtains $D_{T}^{\theta \approx 0}=$ $\frac{1}{2}([a]+[b])$ or $D_{T}^{\theta \approx 0}=\frac{1}{2}([c]+[d])$ or $D_{T}^{\theta \approx 0}=\frac{1}{2}([e]+[f])$, depending on whether one uses $j=f$ or $j=t$ or $j=b$ in Eq. (51c). Similarly, regarding expressions $[g]$ and $[h]$, which are equivalent, Eq. (29) directly leads to $D_{T}^{\theta \approx 0}=\frac{1}{2}([g]+[h])$ when using $j=f$ in Eq. (51c), with equivalent expressions (not shown) when using $j=t$ or $j=b$ in Eq. (51c). Since the same applies to expressions $[i]$ and $[j]$, these examples demonstrate that the diffusivity can typically be described by a number of equivalent expressions when $S>2$.

Although we restrict ourselves to the presentation of diffusivity formulas for 2D landscapes, Eq. (29) is completely general and can be applied to 3D problems as well, provided that all hops are symmetric, as defined in Sec. II A. See the companion report [62] for a complementary method to determine the diffusivity in systems where asymmetric hops are present. The expressions listed in Tables I, III, and IV have been derived using Eq. (29) and confirmed by applying the more general method described in the next part of the present study [62].

\section{NUMERICAL VALIDATION}

Based on the popularity of KMC simulations for determining tracer diffusivities [1-5,51,53,63], we now compare the values obtained from the previous formulas and those determined by KMC simulations. We assume a typical latticegas model, where the adsorption sites form a periodic array and the temperature is sufficiently low so that the adsorbates are well localized around the sites, occasionally hopping between them at rates that are much lower than the typical vibrational frequencies.

As described in Figs. 4(a) and 4(b), we perform two types of simulations. In the first type (KMC-1) the tracer is followed until it hits the perimeter of a circle of radius $R_{o} \gg l_{i j}$, repeating the process for $N_{\mathrm{RW}}$ different random walks (RWs) in order to obtain an ensemble average of the time $\langle t\rangle$ required to cover that distance, thus determining the diffusivity as $D_{T}^{\theta \approx 0}=\frac{1}{2 \alpha} \frac{R_{o}^{2}}{\langle t\rangle}$. In the second type of simulations (KMC-2) the tracer is followed until it performs a desired number of hops $N_{H}$, repeating the process for $N_{\mathrm{RW}}$ different RWs to determine the average squared distance $\left\langle X^{2}+Y^{2}\right\rangle$ covered by the tracer and the corresponding average time $\langle t\rangle$, obtaining the diffusivity by using $D_{T}^{\theta \approx 0}=\frac{1}{2 \alpha} \frac{\left\langle X^{2}+Y^{2}\right\rangle}{\langle t\rangle}$. In both $\mathrm{KMC}-1$ and KMC-2 we perform $G$ ensemble averages (each over $N_{\mathrm{RW}}$ random walks) and report the simulated diffusivity values as the mean \pm standard deviation of the $G$ measurements. Both KMC implementations use the timedependent approach (or variable time stepping), where the next hop (from site $i$ to site $j$ ) is selected with probability $\mu_{i j} / R$, where $R=\Sigma_{i}\left(\Sigma_{j} \mu_{i j}\right)$ is the total rate for all currently accessible jumps, and the corresponding time increment is $\Delta t=-\frac{\log (e)}{R}$, where $e \in(0,1]$ is a uniform random number. At the low-coverage limit there is only one adparticle (currently at site $i$ ), and thus, $R=R_{i}=\Sigma_{k} \mu_{i k}$. For KMC-1, we use $R_{o}=100 l$, where $l$ is the smallest hop length, $N_{\mathrm{RW}}=10^{3}$, and $G=500$. For KMC-2, we use $N_{H}=10^{4}, N_{\mathrm{RW}}=10^{3}$, and $G=100$.

Since the goal is to check the validity of the analytical expressions for the diffusivity, different hop rate values are used to probe situations where the rates have similar values or differ by several orders of magnitude. We also use realistic hop rates for several adsorbates, including $\mathrm{Cu}, \mathrm{Ag}, \mathrm{Rb}$, and $\mathrm{Se}$ on the $\mathrm{Bi}_{2} \mathrm{Se}_{3}(0001)$ surface and in the vdW gap of this material. The hop rates for these physically meaningful examples are obtained by determining the energy barriers through laborintensive DFT calculations (see Sec. IV) and estimating the attempt frequencies by the method described in Figs. 4(c)-4(e). In other words, we make the approximation

$$
v_{o} \approx v_{L}^{A} \approx \frac{1}{2 d} \sqrt{\frac{E_{a}}{2 m}}
$$

where $E_{a}$ is the energy barrier for the hop, $d$ is the separation between the initial site $A$ and the saddle point $T$ (transition state), and $m$ is the adatom mass.

The estimate in Eq. (73) can be considered an alternative to (i) the typical assumption of equal prefactors for all hop rates [1,63-66] and (ii) the large computational cost to determine all the vibrational mode frequencies $v_{i}^{A}$ and $v_{i}^{T}$ for the adatom and substrate at the initial and saddle configurations using 
TABLE II. Simulated and calculated low-coverage diffusivities $\left(D_{T}^{\theta \approx 0} ; \mathrm{cm}^{2} s^{-1}\right)$ for various combinations of hop rates in systems with triangular and rectangular symmetry, including the diffusion of $\mathrm{Cu}, \mathrm{Ag}, \mathrm{Rb}$, and $\mathrm{Se}$ on the $\mathrm{Bi}_{2} \mathrm{Se}_{3}(0001)$ surface (surf) and in the $\mathrm{Bi}_{2} \mathrm{Se}_{3}$ van der Waals gap (vdW) at $T=295 \mathrm{~K}$. Boldface values: Activation energies obtained by DFT.

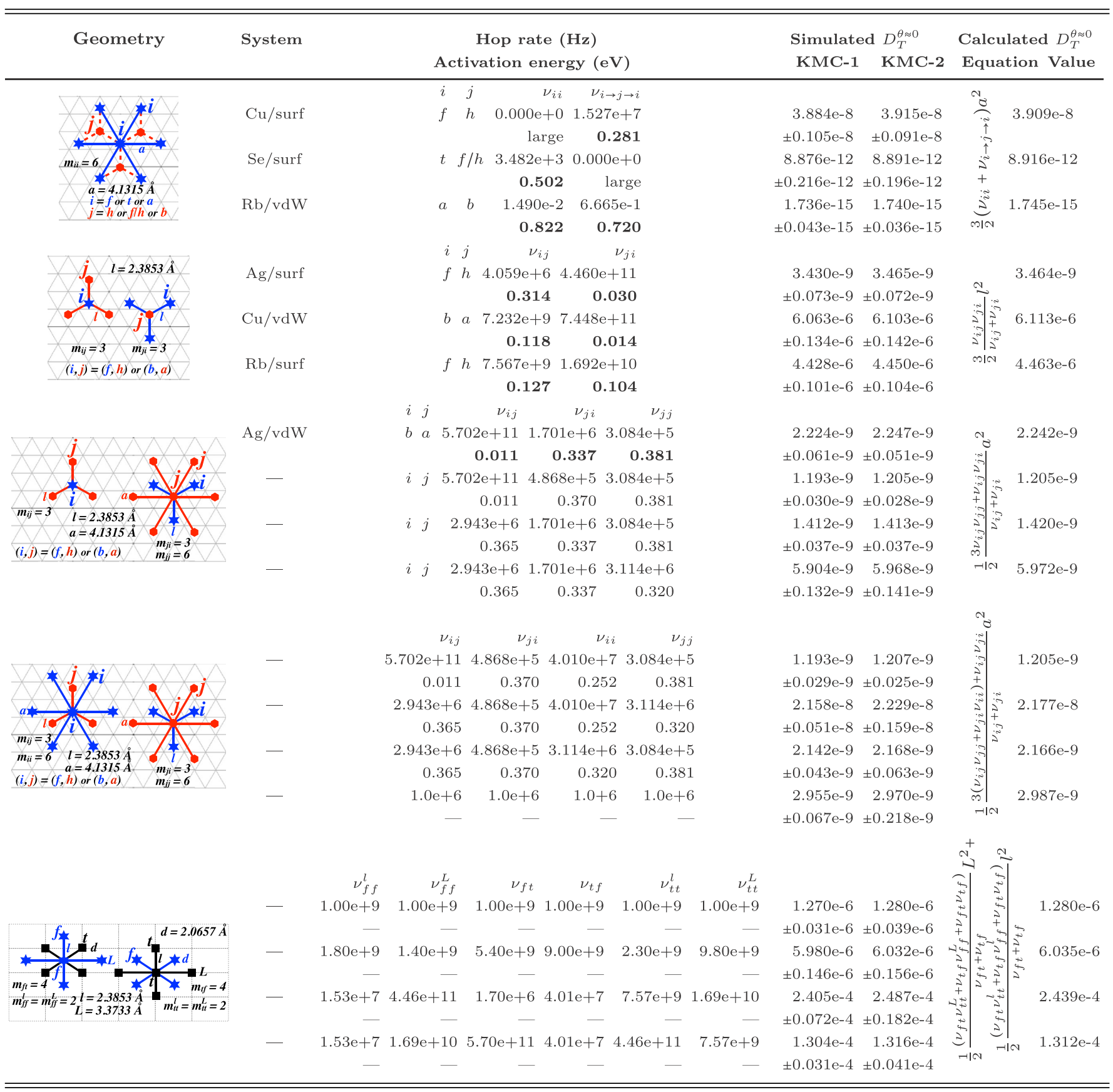

DFT methods [1,64-66] [see expression $U$ for the attempt frequency in Fig. 4(d)]. Due to typical cancellations of the vibrational modes of the substrate [66], the prefactor $v_{o}$ is usually approximated by the Vineyard equation [expression $\mathrm{V}$ in Fig. 4(d)]. Owing to compensation effects between the surface-parallel $\left(v_{\|}^{A}\right.$ and $\left.v_{\|}^{T}\right)$ and the surface-normal $\left(v_{\perp}^{A}\right.$ and $v_{\perp}^{T}$ ) vibrational frequencies of the diffusing atom [66], the Vineyard formula is approximated by just keeping the frequency of the longitudinal vibrations (along the diffusion path) of the atom at the initial site $\left[v_{L}^{A}\right.$; see expression $\mathrm{W}$ in Fig. 4(d)]. Our estimate consists of approximating the longitudinal path by a sinusoidal path, resulting in a simple and physically meaningful expression for $v_{L}^{A}$ in terms of the energy barrier, hop distance, and adatom mass, as described in Fig. 4(f) and contained in Eq. (73). Although the actual energy path can be asymmetric with respect to the saddle point $T$, the part after this point is irrelevant for the rate calculation and in our approximation it is considered to be a reflection of the part before it.

Table II provides a comparison of the calculated values for the low-coverage tracer diffusivities according to the derived formulas and those obtained from the KMC simulations for 


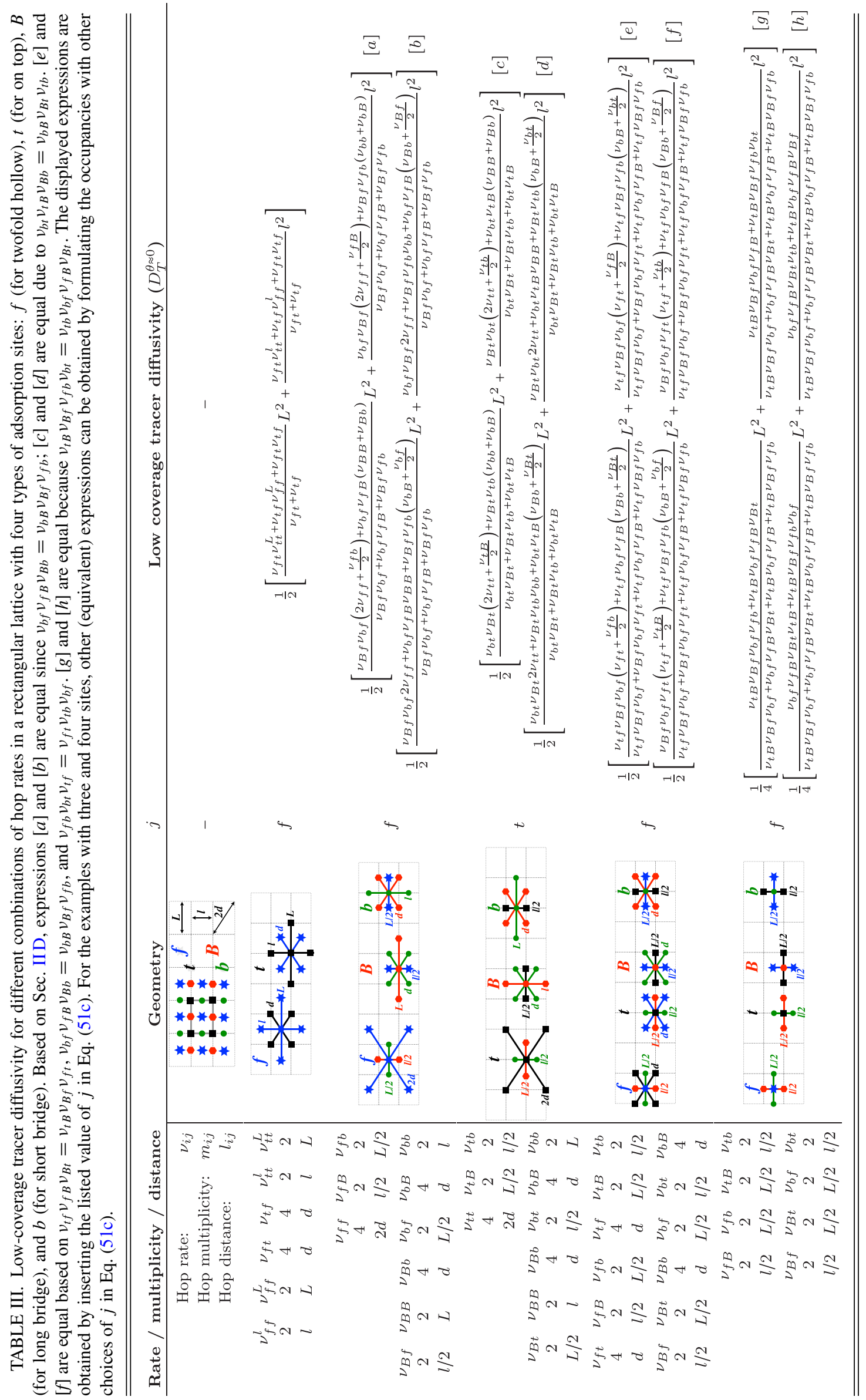



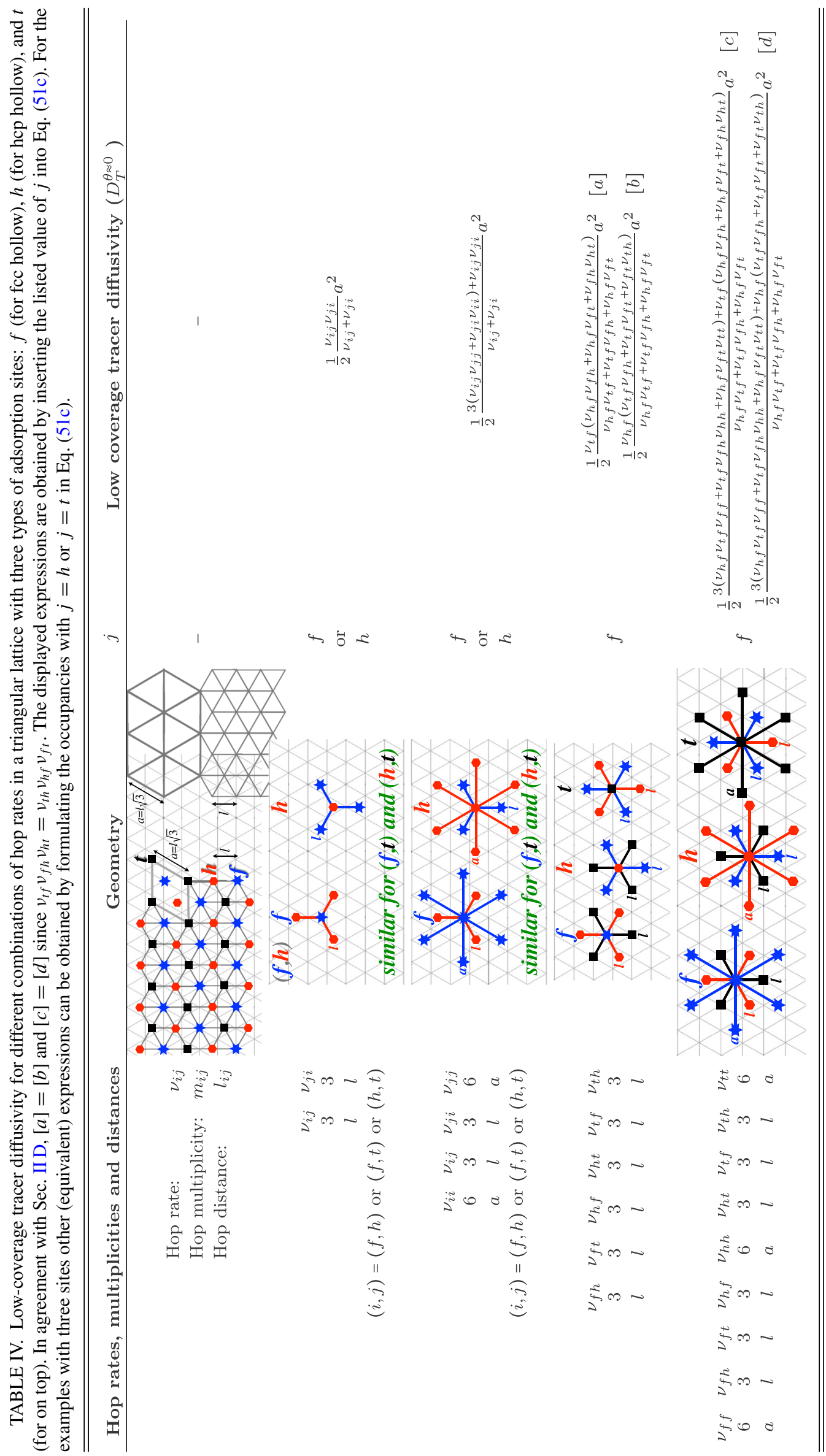


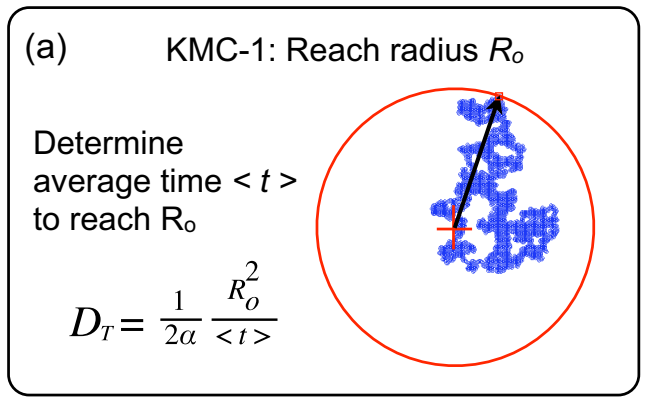

(b) KMC-2: Reach number of hops $N_{H}$

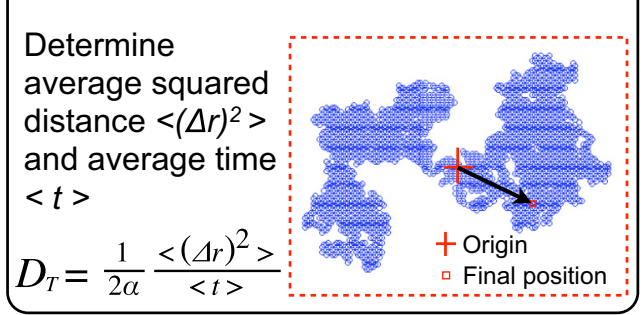

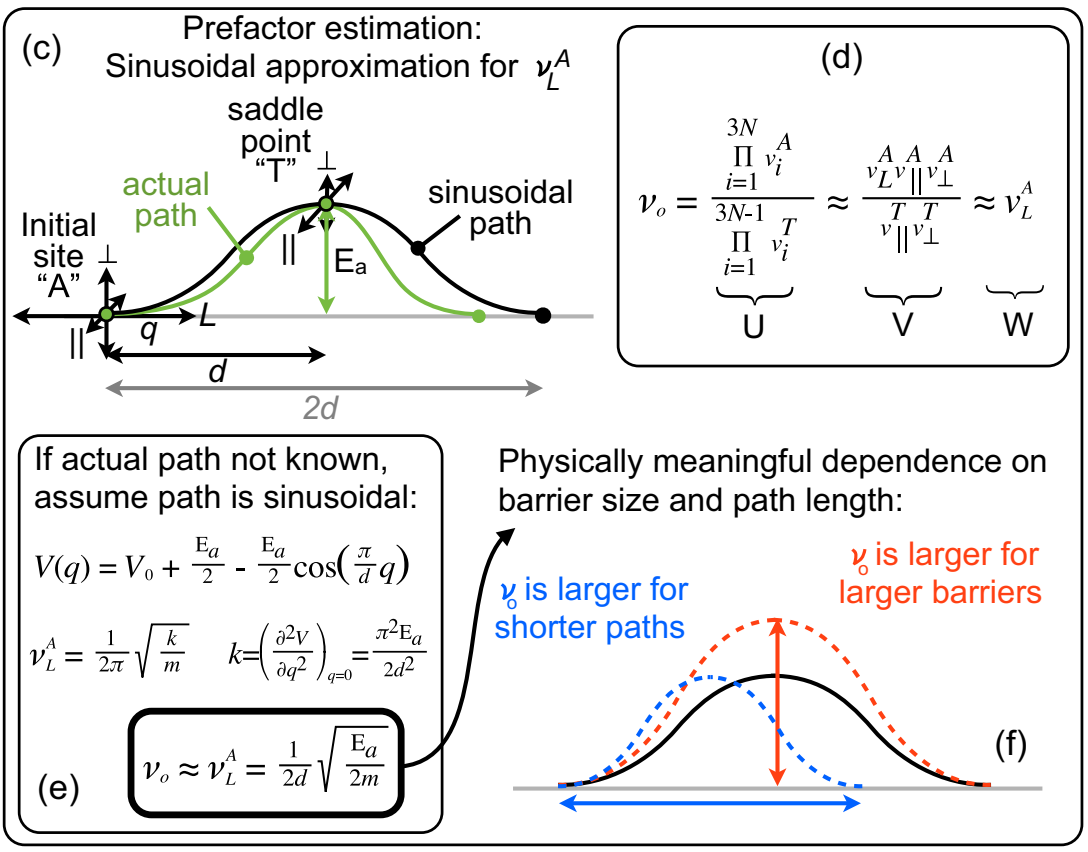

FIG. 4. Illustration of the two simulation procedures used to perform KMC simulations of diffusion in this study: (a) KMC-1; (b) KMC-2. (c-e) Estimations of the attempt frequencies in this study. (f) Interpretation of the attempt-frequency dependence on the barrier size and path length.

systems with triangular and rectangular symmetry where all hops are symmetric. In this table, the activation energies listed in the third column are used to determine both the Boltzmann factors $\left(e^{-E_{a} / k_{B} T}\right)$ and the prefactors $\left(v_{o}=\sqrt{E_{a} / 2 m} / 2 d\right)$. Those activation energies determined by our DFT calculations are shown in boldface in the table. For hops of types $v_{i j}$, $v_{i i}, v_{j i}$, and $v_{j j}$ the barrier is located in the middle of the straight line between the initial and the final sites. Thus, the factor $2 d$ in the expression for $v_{o}$ is equal to the hop distance: $2 d=l_{i j}, l_{i i}, l_{j i}$, or $l_{j j}$, respectively. For hops of type $v_{i \rightarrow j \rightarrow i}$ the hop distance is $l_{i i}$ but the path is not straight, the energy maximum being located at site $j$. Thus, in this case, $2 d=2 l_{i j} \neq l_{i i}$. All other considered activation energies are used to probe different combinations of hop rates spanning several orders of magnitude for different lattices.

For all considered systems in Table II the simulated and calculated diffusivities agree extremely well with each other. Thus, the proposed formulas are suitable for discussion of the low-coverage tracer diffusivity of typical diffusion species in complex energy landscapes without the need to perform the corresponding KMC simulations.

\section{DFT CALCULATIONS}

In this study the reported diffusion barriers (activation energies) are the result of a large computational effort based on DFT calculations. The calculations are performed using the projector augmented-wave method [67] in the VASP implementation $[68,69]$. The exchange-correlation potential is described by the generalized gradient approximation [70], and the spin-orbit coupling is neglected. Test calculations performed including the latter show that the activation energy changes only a few percent, e.g., 1.1\%, for $\mathrm{Rb}$. To account for the vdW interactions we make use of the DFT-D2 approach proposed by Grimme [71].

The crystal structure of $\mathrm{Bi}_{2} \mathrm{Se}_{3}$ is formed by quintuple layer (QL) blocks separated by the vdW gaps, as shown in Fig. 5(a). In each QL the hexagonal atomic planes follow the order Se$\mathrm{Bi}-\mathrm{Se}-\mathrm{Bi}-\mathrm{Se}$. While the bonding between atoms that belong to the same QL is of the covalent-ionic type, neighboring QLs are only weakly bonded by vdW forces. The diffusion barriers on the surface are determined by using a slab of 5 atomic layers (i.e., 1 QL), as schematically described in Fig. 5(b.1), while the diffusion within the vdW gap is studied using a slab of 10 atomic layers (2 QLs), as described in Fig. 5(b.2). In both cases, essentially isolated adatoms are considered by placing them in the $3 \times 3$ supercell. Figure 5(c) shows a top view of the typical locations of the energy minima (adsorption sites) found in the vdW gap. The behavior of the $\mathrm{Cu}$ and $\mathrm{Ag}$ adatoms in the vicinity of [01 10$]$-oriented ([1120]-oriented) steps is studied using a supercell containing 1 full $3 \times 6(2 \sqrt{3} \times 6)$ QL and $3 \times 3(2 \sqrt{3} \times 3)$ step-terminated QLs on top of it, as shown schematically in Fig. 5(b.3). For Rb adatoms the long side of the supercell is lengthened by a factor of 1.5 (1.333) to better account for the stronger relaxations.

Figure 5(d) provides several diffusion path geometries and energy profiles determined in our DFT calculations for diffusion of $\mathrm{Ag}$ atoms along the terrace, in the vdW gap, and across the step, as described below. Similar results are obtained for all considered adatoms in the same/other terrace/vdW/step geometries.

\section{A. Terrace diffusion}

We start by determining the total energy at locations $f, h$, $t$, and $b$ [see Fig. 2(a)], discarding those locations where the energy is excessively high. Let us refer to the nondiscarded 

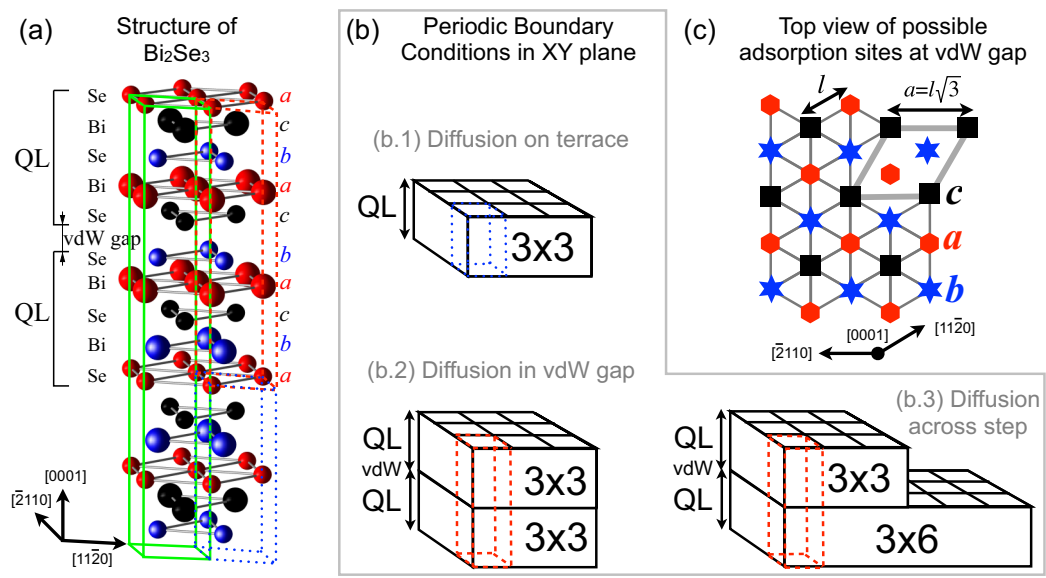
(d) Examples of diffusion path geometries determined by the DFT calculations of this study

(d.1) in vdW (d.2) across step (d.3) on terrace

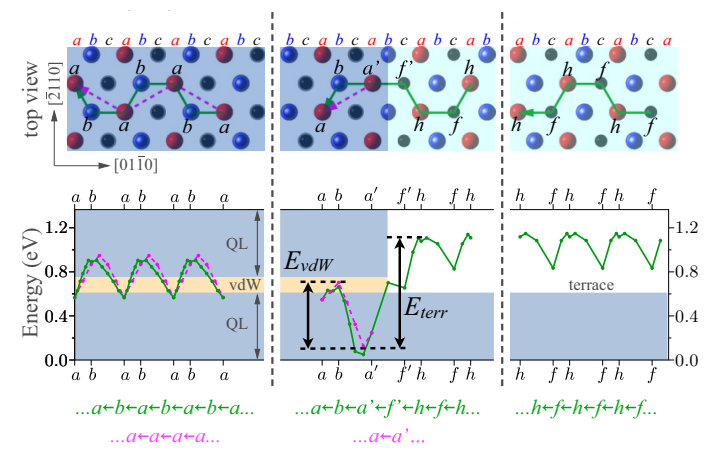

FIG. 5. (a) Crystal structure of $\mathrm{Bi}_{2} \mathrm{Se}_{3}$, showing the $\mathrm{Bi}$ and $\mathrm{Se}$ atoms as large and small spheres, respectively, using different colors according to the stacking sequence: red for $a$, blue for $b$, and black for $c$. (b) Several crystallographic cells are highlighted (solid, dashed, and dotted lines) for cross-reference. (b) Schematic supercell structure used for the calculation of diffusion barriers on the terrace, in the vDW gap, and across steps, as indicated. (c) Top view of possible adsorption sites at the vdW gap: $a, b$, and $c$ are locations aligned vertically with each stack, respectively. (d) Example of diffusion path geometries and energies determined through our DFT calculations for the Ag adatom.

locations as accessible sites. They may correspond to adsorption sites or saddle points, although this is decided later. Due to symmetry, any other possible adsorption site and/or saddle point - if it exists - is necessarily located between neighboring accessible sites. Correspondingly, for any pair of accessible sites we consider the projection (on the $X Y$ plane) of the line that links both sites and proceed to move the adatom incrementally along that projection. After every incremental move, the vertical coordinate of the adatom and all three coordinates of all other atoms are allowed to relax, except for the atoms located at the bottom of the QL, which are kept frozen. In this manner, we sample the diffusion energy along all $X Y$-straight paths between all possible adsorption sites and saddle points. The diffusion barriers (or activation energies) reported in this study correspond to the energy difference between the initial point (always an adsorption site) and the highest energy point along the corresponding path (always a saddle point or absolute maximum). This approach has been verified using the nudged elastic band method [72,73] for the case of $\mathrm{Rb}$ on $\mathrm{Bi}_{2} \mathrm{Se}_{3}(0001)$ (this can also be done using the dimer method [74]). The results obtained are found to be in excellent agreement regarding the location of the saddle point and the activation energy value.

\section{B. Diffusion in the vdW gap}

For diffusion in the vdW gap, we similarly determine the energy at octahedral $(a)$ and tetrahedral ( $b$ and $c$ ) positions [see Fig. 5(c)] in order to resolve which are the accessible sites. We then proceed as in the case for terrace diffusion in order to determine the diffusion paths, their energy profiles, and the corresponding diffusion barriers.

\section{Diffusion across the steps}

For diffusion across the steps, the number of possibilities increases due to the different step orientations, [1120] and [0110], the latter having two possible atomic terminations. As an example, Fig. 5(d) considers the [0110]-type I step geometry for the case of Ag adatoms. A DFT study of diffusion within the vdW gap [Fig. 5(d.1)] concludes that only the $a$ sites can be considered strong adsorption sites. Similarly, a terrace diffusion study [Fig. 5(d.3)] infers that the $f$ and $h$ sites act as strong adsorption centers in this case. The diffusion of the adatom across the step is studied by (i) starting at a surface site that is most distant (within the supercell) from the step [ $h$ in Fig. 5(d.2)], (ii) moving the adatom towards the step along a previously determined terrace diffusion path $[h \rightarrow f \rightarrow h \rightarrow$ $f^{\prime}$ in Fig. 5(d.2)], (iii) crossing the step by linking site $f^{\prime}$ (an intermediate $f$-like site located in the proximity of the step) and site $a^{\prime}$ (an intermediate $a$-like site in the opposite proximity of the step), and (iv) moving the adatom away from the step along a previously determined vdW diffusion path [ $a^{\prime} \rightarrow b \rightarrow a$ in Fig. 5(d.2)], ending at a vdW site that is most distant (within the supercell) from the step [ $a$ in Fig. 5(d.2)]. The study proceeds by performing similar calculations for the other step geometries, namely, the [0110]-type II and [1120] steps. This allows the determination of the diffusion barrier for penetration into the $\mathrm{vdW}$ gap $\left(E_{\mathrm{vdW}}\right)$ and reentry into the terrace region $\left(E_{\text {terr }}\right)$ for the three step geometries. For each geometry, if more than one energy path across the step is determined, as in Fig. 5(d.2), $E_{\mathrm{vdW}}$ and $E_{\text {terr }}$ are reported as the minimum values among those paths.

\section{APPLICATION TO TOPOLOGICAL INSULATORS}

Encouraged by the validation of the diffusivity formulas we now consider the temperature dependence of the diffusion of $\mathrm{Cu}, \mathrm{Ag}$, and $\mathrm{Rb}$ adatoms on the $\mathrm{Bi}_{2} \mathrm{Se}_{3}(0001)$ surface and the corresponding intercalation of these adsorbates in the $\mathrm{Bi}_{2} \mathrm{Se}_{3} \mathrm{vdW}$ gap. Figures 6(a) and 6(b) provide the calculated diffusion length $\Lambda=\sqrt{2 \alpha D_{T}^{\theta \approx 0} t}$ as a function of the temperature for the three considered adatoms when they diffuse on the surface and in the vdW gap, respectively. The evaluations have been done on the basis of the formulas obtained for $D_{T}^{\theta \approx 0}$ (see final column in Table II). For diffusion with a single barrier $E_{a}$, the underlying assumption that the 


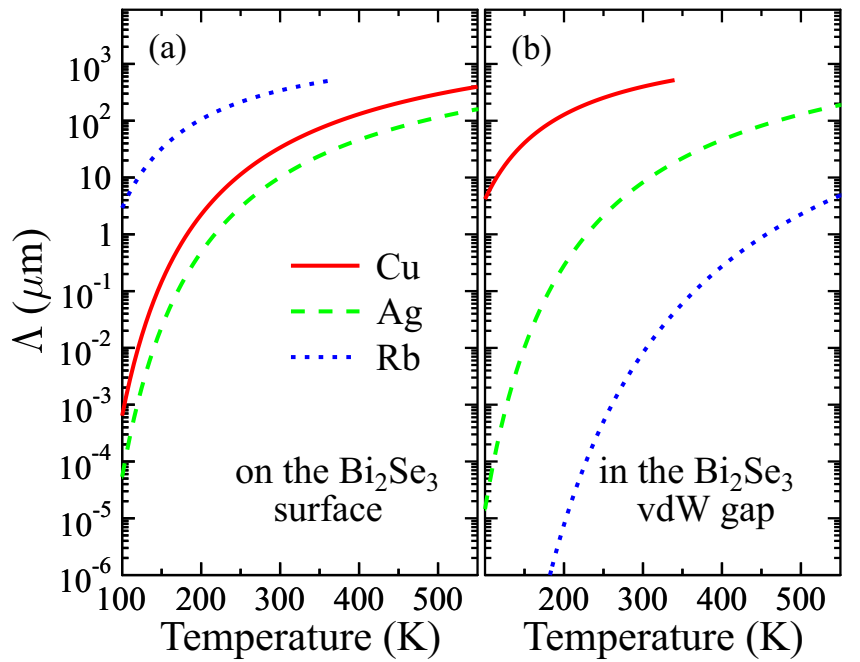

FIG. 6. Calculated diffusion length $\Lambda$ (in logarithmic scale) as a function of temperature for $\mathrm{Cu}, \mathrm{Ag}$, and $\mathrm{Rb}$ adatoms: (a) on the $\mathrm{Bi}_{2} \mathrm{Se}_{3}(0001)$ surface and (b) in the $\mathrm{Bi}_{2} \mathrm{Se}_{3}$ vdW gap. Diffusion time: $1 \mathrm{~min}$.

hops can be treated as rare events (compared to the fast vibrations around the adsorption sites) is valid if $E_{a}>4 k_{B} T$ (see Ref. [1]). Correspondingly, each displayed curve in Fig. 6 terminates at $T_{\max }=E_{a, \max } / 4 k_{B}$, where $E_{a, \text { max }}$ is the maximum barrier experienced by the corresponding adatom. As an example, the diffusion of $\mathrm{Rb}$ on the surface experiences two barriers: $E_{f h}=0.127 \mathrm{eV}$ and $E_{h f}=0.104 \mathrm{eV}$. Thus, $E_{a, \max }=0.127 \mathrm{eV}$ and $T_{\max }=368 \mathrm{~K}$. Similarly, for $\mathrm{Cu}$ in the vdW gap we have $T_{\max }=342 \mathrm{~K}$. Nevertheless, the total temperature range is restricted to $550 \mathrm{~K}$ since the desorption of $\mathrm{Cu}$ is reported to start approximately at this temperature [75].

It is shown in Fig. 6 that the hierarchy of the diffusion length is $\Lambda_{\mathrm{Rb}}>\Lambda_{\mathrm{Cu}}>\Lambda_{\mathrm{Ag}}$ on the surface and $\Lambda_{\mathrm{Cu}}>\Lambda_{\mathrm{Ag}}>\Lambda_{\mathrm{Rb}}$ in the vdW gap. The $\mathrm{Rb}(\mathrm{Cu})$ atoms are the most mobile species on the surface (in the vdW gap), capable of covering more than $1 \mu \mathrm{m}$ within $1 \mathrm{~min}$ even at $100 \mathrm{~K}$. However, the $\mathrm{vdW}$ (surface) diffusion length of the $\mathrm{Rb}(\mathrm{Cu})$ atoms is much lower than that on the surface (in the vdW), which is due to the significantly higher diffusion barriers. Interestingly, the Ag atoms travel at almost-equal rates on the surface and in the vdW gap.

Let us now bring the discussion closer to the available experiments $[23,25,26]$. These indicate indirectly the occurrence of partial [23] or almost-complete [25,26] intercalation of the metal adatoms inside the $\mathrm{Bi}_{2} \mathrm{Se}_{3} \mathrm{vdW}$ gap at room and higher temperatures. Recently, it has been argued [31] that the intercalation of the metal atoms in the $\mathrm{Bi}_{2} \mathrm{Se}_{3} \mathrm{vdW}$ gap is step mediated, in the sense that they penetrate into the vdW gap after reaching the steps, which are typically present at the $\mathrm{Bi}_{2} \mathrm{Se}_{3}$ surface [25]. This is favored by the geometrical alignment between the terrace and the vdW gap in a stepped surface, as schematically shown in Fig 7(a). At the same time, penetration of $\mathrm{Cu}$ [25] and $\mathrm{Ag}$ [31] in the vdW gap via interstitials and/or vacancies of the topmost $\mathrm{Bi}_{2} \mathrm{Se}_{3} \mathrm{QL}$ is significantly less probable due to high-energy barriers. Therefore, one may also rule out the possibility of vertical penetration of the $\mathrm{Rb}$ atom, whose covalent radius is $1.5(1.66)$ times larger than that
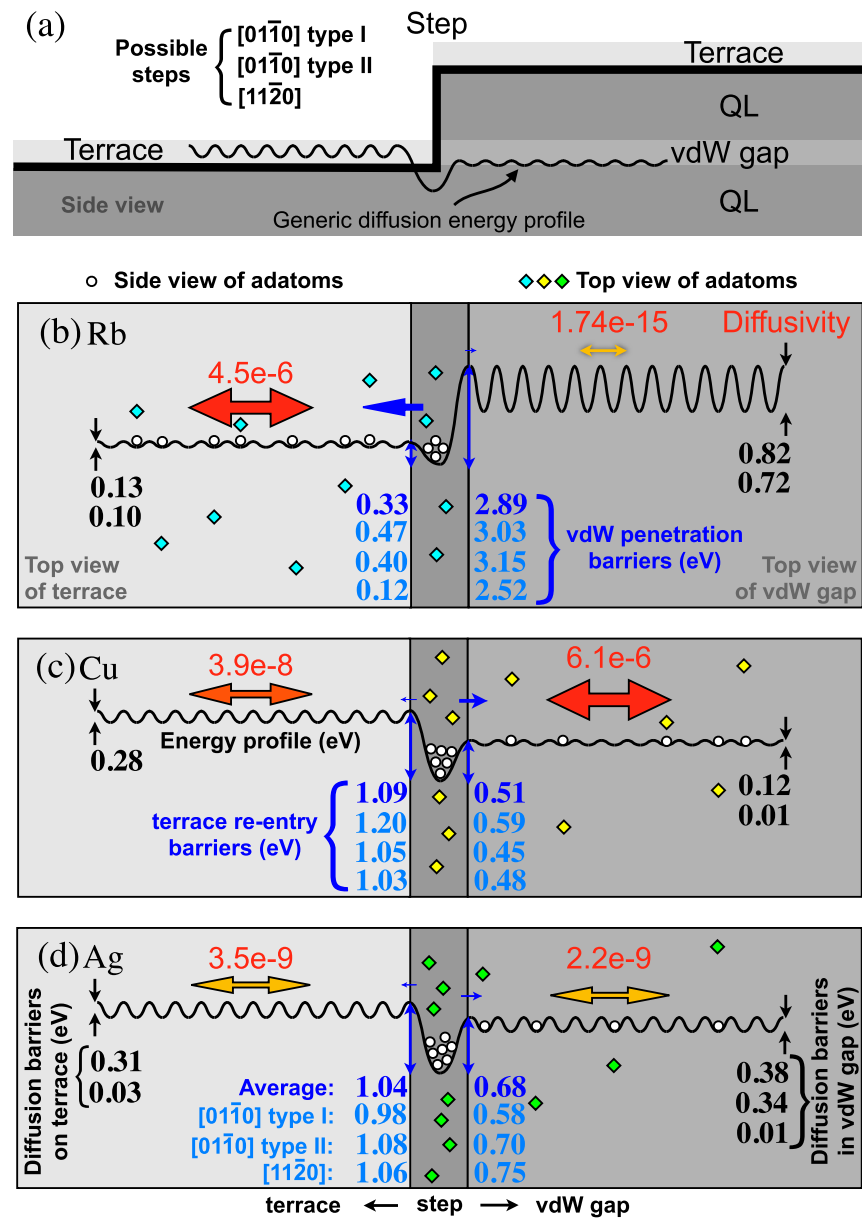

FIG. 7. (a) Geometrical alignment between terrace and vdW gap at a morphological step. (b-d) Schematic of the low-coverage diffusivity (in $\mathrm{cm}^{2} \mathrm{~s}^{-1}$ ) of $\mathrm{Rb}, \mathrm{Cu}$, and $\mathrm{Ag}$ on the terrace and in the vdW gap of $\mathrm{Bi}_{2} \mathrm{Se}_{3}$ at room temperature. Larger arrows denote larger diffusivities. Our DFT-calculated diffusion barriers (in eV) are shown in boldface for (left) terrace diffusion, (center left) terrace reentry, (center right) vdW-gap penetration, and (right) vdW-gap diffusion.

of $\mathrm{Ag}(\mathrm{Cu})$, whereupon the diffusion barriers are expected to be even higher.

By using large and small double-headed arrows, Figs. 7(b)7 (d) present a graphical description of the relative diffusivity of the three types of adatoms on the terraces and within the vdW gaps. In addition, Fig. 7 also provides the relative rates to enter the vdW gap and to bounce back to the terrace by assigning them large/small unidirectional arrows. Moreover, the figure collects all the diffusion barriers determined by our DFT calculations for the three adatoms on the terrace and in the vdW gap, as well as for their penetration into the vdW gap and reentry into the terrace for two step orientations,

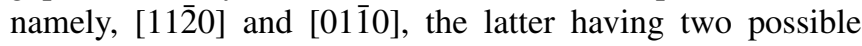
atomic terminations [31]. To ease the discussion, the three barriers for $\mathrm{vdW}$ penetration (terrace reentry) for each adatom are algebraically averaged and used to estimate the $\mathrm{vdW}$ penetration (terrace reentry) rate of that atom, accordingly assigning them large/small unidirectional arrows. We focus the discussion on room temperature $(295 \mathrm{~K})$ and long diffusion times. 
The $\mathrm{Rb}$ atoms have the highest terrace and lowest vdW diffusivities, with a very low vdW penetration rate and a rather high terrace reentry rate. Thus, the $\mathrm{Rb}$ atoms are expected to quickly diffuse across the terraces and hit the steps, where a small fraction will remain trapped while the majority will bounce back to the terraces. This is described schematically in Fig. 7(b), where a large number of $\mathrm{Rb}$ atoms are drawn in the terrace region, with additional atoms at the step, and hardly any atoms in the vdW gap. In comparison, the $\mathrm{Cu}$ atoms are characterized by moderate terrace and highest vdW diffusivities, with the highest vdW penetration rate and a low terrace reentry rate. Accordingly, the $\mathrm{Cu}$ atoms need a longer time to arrive at the steps but they eventually penetrate with relative ease into the $\mathrm{vdW}$ gap, where they diffuse rather rapidly. Thus, the $\mathrm{Cu}$ atoms are expected to mostly intercalate in the vdW gap, although a notable fraction will remain at the steps, as sketched in Fig. 7(c). Finally, the Ag atoms display a slightly lower terrace diffusivity compared to $\mathrm{Cu}$ and a medium diffusivity in the vdW gap among the three species under consideration. Having reached the steps, the Ag atoms are forced to linger along them due to the large terrace reentry barrier and significant vdW penetration barrier [Fig. 7(d)]. Since the latter is smaller, the $\mathrm{Ag}$ atoms are expected to gradually intercalate into the vdW gap. The behavior is similar to that of $\mathrm{Cu}$, although intercalation is slower for $\mathrm{Ag}$. Valid only for the low-coverage limit, these trends are in good qualitative agreement with those from available experiments $[23,25,26]$.

\section{CONCLUSIONS}

This article initiates a series of two papers dedicated to the presentation of a general expression to calculate the singleparticle diffusion coefficient in any number of dimensions for systems with complex, periodic energy landscapes, where the end sites of the hops can be located symmetrically (this paper) and/or asymmetrically [62] with respect to the hop origins. In particular, the present paper introduces a simple, general expression to determine the average motion of the diffusing particle in systems that contain symmetric hops only, focusing then on the analysis of adsorbate diffusion on surfaces and within the 2D gaps of layered materials with triangular, rectangular, and square symmetry, finally applying the resulting formulas to discuss intercalation in a model topological insulator, $\mathrm{Bi}_{2} \mathrm{Se}_{3}$. The analytical expressions are validated against KMC simulations, obtaining excellent agreement between calculated and simulated diffusivities. Thus, one can determine the overall diffusion coefficient without performing the KMC simulations.

Based on diffusion rates obtained from energy barriers procured by labor-intensive DFT calculations, we analyze the temperature dependence of the diffusion of $\mathrm{Cu}, \mathrm{Ag}$, and $\mathrm{Rb}$ on the $\mathrm{Bi}_{2} \mathrm{Se}_{3}(0001)$ surface and within the vdW gap of this layered material. We also analyze the occurrence of adsorbate intercalation due to the alignment between vdW gaps and terraces on the stepped (0001) surface of this topological insulator. At room temperature and low coverage, we conclude that the $\mathrm{Rb}$ atoms quickly diffuse across the terraces and hit the steps, where a small number remain trapped while the rest bounce back into the terraces. Thus, $\mathrm{Rb}$ is expected to partially decorate the steps while remaining present on the terraces. In comparison, the $\mathrm{Cu}$ atoms take a longer time to arrive at the steps, eventually penetrating with relative ease into the vdW gap. Thus, $\mathrm{Cu}$ atoms are expected to mostly intercalate into the vdW gap while partially remaining at the steps. Ag atoms are expected to take an even longer time to diffuse across the terraces, eventually penetrating into the vdW gap with time but meanwhile remaining around the steps.

\section{ACKNOWLEDGMENTS}

We acknowledge support from the Ramón y Cajal Fellowship Program of the Spanish Ministry of Science and Innovation (M. A. Gosálvez), the JAE-Doc grant from the 'Junta para la Ampliación de Estudios' program cofunded by FSE (N. Ferrando), the University of the Basque Country (Grant No. GIC07IT36607), the Spanish Ministry of Science and Innovation (Grant Nos. FIS2013-48286-C02-02-P and FIS2013-48286-C02-01-P), the Basque Government through the Nanomaterials project under the nanoGUNE2014 program (Grant No. IE05-151), the Tomsk State University Academic D. I. Mendeleev Fund Program in 2015 (Research Grant No. 8.1.05.2015), and partial support from Saint Petersburg State University (Project No. 15.61.202.2015). The DFT calculations were performed on the SKIF-Cyberia supercomputer at Tomsk State University and at the Donostia International Physics Center.
[1] T. Ala-Nissila, R. Ferrando, and S. Ying, Adv. Phys. 51, 949 (2002).

[2] A. Naumovets and Z. Zhang, Surf. Sci. 500, 414 (2002).

[3] R. Gomer, Rep. Prog. Phys. 53, 917 (1990).

[4] G. L. Kellogg, in Encyclopedia of Materials: Science and Technology (Elsevier, Amsterdam, 2001), pp. 9025-9030.

[5] R. Ferrando, in Encyclopedia of Materials: Science and Technology (Elsevier, Amsterdam, 2006), pp. 1-6.

[6] Z. Ma and F. Zaera, Encyclopedia of Inorganic Chemistry (John Wiley \& Sons, New York, 2006).

[7] X. Li, W. Cai, J. An, S. Kim, J. Nah, D. Yang, R. Piner, A. Velamakanni, I. Jung, E. Tutuc, S. K. Banerjee, L. Colombo, and R. S. Ruoff, Science 324, 1312 (2009).
[8] S. Bae, H. Kim, Y. Lee, X. Xu, J.-S. Park, Y. Zheng, J. Balakrishnan, T. Lei, H. R. Kim, Y. I. Song, Y.-J. Kim, K. S. Kim, B. Ozyilmaz, J.-H. Ahn, B. H. Hong, and S. Iijima, Nat. Nanotechnol. 5, 574 (2010).

[9] F. Calleja, H. Ochoa, M. Garnica, S. Barja, J. J. Navarro, A. Black, M. M. Otrokov, E. V. Chulkov, A. Arnau, A. L. V. de Parga, F. Guinea, and R. Miranda, Nat. Phys. 11, 43 (2015).

[10] F. Bonaccorso, A. Lombardo, T. Hasan, Z. Sun, L. Colombo, and A. C. Ferrari, Mater. Today 15, 564 (2012).

[11] D. Usachov, A. Fedorov, M. M. Otrokov, A. Chikina, O. Vilkov, A. Petukhov, A. G. Rybkin, Y. M. Koroteev, E. V. Chulkov, V. K. Adamchuk, A. Grüneis, C. Laubschat, and D. V. Vyalikh, Nano Lett. 15, 2396 (2015). 
[12] D. Y. Usachov, A. V. Fedorov, A. E. Petukhov, O. Y. Vilkov, A. G. Rybkin, M. M. Otrokov, A. Arnau, E. V. Chulkov, L. V. Yashina, M. Farjam, V. K. Adamchuk, B. V. Senkovskiy, C. Laubschat, and D. V. Vyalikh, ACS Nano 9, 7314 (2015).

[13] M. Xu, T. Liang, M. Shi, and H. Chen, Chem. Rev. 113, 3766 (2013).

[14] H. Wang, L. Yu, Y. Lee, W. Fang, A. Hsu, P. Herring, M. Chin, M. Dubey, L. Li, J. Kong, and T. Palacios, in Electron Devices Meeting (IEDM), 2012 IEEE International (IEEE, Piscataway, NJ, 2012), pp. 4.6.1-4.6.4.

[15] Y.-H. Lee, X.-Q. Zhang, W. Zhang, M.-T. Chang, C.-T. Lin, K.-D. Chang, Y.-C. Yu, J. T.-W. Wang, C.-S. Chang, L.-J. Li, and T.-W. Lin, Adv. Mater. 24, 2320 (2012).

[16] A. Ismach, H. Chou, D. A. Ferrer, Y. Wu, S. McDonnell, H. C. Floresca, A. Covacevich, C. Pope, R. Piner, M. J. Kim, R. M. Wallace, L. Colombo, and R. S. Ruoff, ACS Nano 6, 6378 (2012).

[17] L. Ci, L. Song, C. Jin, D. Jariwala, D. Wu, Y. Li, A. Srivastava, Z. F. Wang, K. Storr, L. Balicas, F. Liu, and P. M. Ajayan, Nat. Mater. 9, 430 (2010).

[18] N. D. Boscher, C. J. Carmalt, and I. P. Parkin, Appl. Surf. Sci. 256, 3178 (2010).

[19] Y. Yan, Z.-M. Liao, Y.-B. Zhou, H.-C. Wu, Y.-Q. Bie, J.-J. Chen, J. Meng, X.-S. Wu, and D.-P. Yu, Sci. Rep. 3, 1264 (2013).

[20] H. Li, J. Cao, W. Zheng, Y. Chen, D. Wu, W. Dang, K. Wang, H. Peng, and Z. Liu, J. Am. Chem. Soc. 134, 6132 (2012).

[21] M. Bianchi, R. C. Hatch, J. Mi, B. B. Iversen, and P. Hofmann, Phys. Rev. Lett. 107, 086802 (2011).

[22] Z.-H. Zhu, G. Levy, B. Ludbrook, C. N. Veenstra, J. A. Rosen, R. Comin, D. Wong, P. Dosanjh, A. Ubaldini, P. Syers, N. P. Butch, J. Paglione, I. S. Elfimov, and A. Damascelli, Phys. Rev. Lett. 107, 186405 (2011).

[23] M. Bianchi, R. C. Hatch, Z. Li, P. Hofmann, F. Song, J. Mi, B. B. Iversen, Z. M. Abd El-Fattah, P. Loeptien, L. Zhou, A. A. Khajetoorians, J. Wiebe, R. Wiesendanger, and J. W. Wells, ACS Nano 6, 7009 (2012).

[24] T. Valla, Z.-H. Pan, D. Gardner, Y. S. Lee, and S. Chu, Phys. Rev. Lett. 108, 117601 (2012).

[25] Y.-L. Wang, Y. Xu, Y.-P. Jiang, J.-W. Liu, C.-Z. Chang, M. Chen, Z. Li, C.-L. Song, L.-L. Wang, K. He, X. Chen, W.H. Duan, Q.-K. Xue, and X.-C. Ma, Phys. Rev. B 84, 075335 (2011).

[26] M. Ye, S. V. Eremeev, K. Kuroda, M. Nakatake, S. Kim, Y. Yamada, E. E. Krasovskii, E. V. Chulkov, M. Arita, H. Miyahara, T. Maegawa, K. Okamoto, K. Miyamoto, T. Okuda, K. Shimada, H. Namatame, M. Taniguchi, Y. Ueda, and A. Kimura, arXiv:1112.5869.

[27] M. Ye, S. V. Eremeev, K. Kuroda, E. E. Krasovskii, E. V. Chulkov, Y. Takeda, Y. Saitoh, K. Okamoto, S. Y. Zhu, K. Miyamoto, M. Arita, M. Nakatake, T. Okuda, Y. Ueda, K. Shimada, H. Namatame, M. Taniguchi, and A. Kimura, Phys. Rev. B 85, 205317 (2012).

[28] M. R. Scholz, J. Sánchez-Barriga, D. Marchenko, A. Varykhalov, A. Volykhov, L. V. Yashina, and O. Rader, Phys. Rev. Lett. 108, 256810 (2012).

[29] C. Seibel, H. Maaß, M. Ohtaka, S. Fiedler, C. Jünger, C.-H. Min, H. Bentmann, K. Sakamoto, and F. Reinert, Phys. Rev. B 86, 161105 (2012).
[30] S. V. Eremeev, M. G. Vergniory, T. V. Menshchikova, A. A. Shaposhnikov, and E. V. Chulkov, New J. Phys. 14, 113030 (2012).

[31] M. M. Otrokov, S. D. Borisova, V. Chis, M. G. Vergniory, S. V. Eremeev, V. M. Kuznetsov, and E. V. Chulkov, JETP Lett. 96, 714 (2013).

[32] T. Eelbo, M. Sikora, G. Bihlmayer, M. Dobrzański, A. Kozłowski, I. Miotkowski, and R. Wiesendanger, New J. Phys. 15, 113026 (2013)

[33] S. Roy, H. L. Meyerheim, A. Ernst, K. Mohseni, C. Tusche, M. G. Vergniory, T. V. Menshchikova, M. M. Otrokov, A. G. Ryabishchenkova, Z. S. Aliev, M. B. Babanly, K. A. Kokh, O. E. Tereshchenko, E. V. Chulkov, J. Schneider, and J. Kirschner, Phys. Rev. Lett. 113, 116802 (2014).

[34] S. Roy, H. L. Meyerheim, K. Mohseni, A. Ernst, M. M. Otrokov, M. G. Vergniory, G. Mussler, J. Kampmeier, D. Grützmacher, C. Tusche, J. Schneider, E. V. Chulkov, and J. Kirschner, Phys. Rev. B 90, 155456 (2014).

[35] R. Shokri, H. L. Meyerheim, S. Roy, K. Mohseni, A. Ernst, M. M. Otrokov, E. V. Chulkov, and J. Kirschner, Phys. Rev. B 91, 205430 (2015).

[36] A. Polyakov, H. L. Meyerheim, E. D. Crozier, R. A. Gordon, K. Mohseni, S. Roy, A. Ernst, M. G. Vergniory, X. Zubizarreta, M. M. Otrokov, E. V. Chulkov, and J. Kirschner, Phys. Rev. B 92, 045423 (2015).

[37] A. G. Ryabishchenkova, M. M. Otrokov, V. M. Kuznetsov, and E. V. Chulkov, J. Exp. Theor. Phys. 121, 465 (2015).

[38] N. de Jong, E. Frantzeskakis, B. Zwartsenberg, Y. K. Huang, D. Wu, P. Hlawenka, J. Sánchez-Barriga, A. Varykhalov, E. van Heumen, and M. S. Golden, Phys. Rev. B 92, 075127 (2015).

[39] M. M. Otrokov, E. V. Chulkov, and A. Arnau, Phys. Rev. B 92, 165309 (2015)

[40] Y. Hor, J. Checkelsky, D. Qu, N. Ong, and R. Cava, J. Phys. Chem. Solids 72, 572 (2011).

[41] K. J. Koski, C. D. Wessells, B. W. Reed, J. J. Cha, D. Kong, and Y. Cui, J. Am. Chem. Soc. 134, 13773 (2012).

[42] Y. S. Hor, A. J. Williams, J. G. Checkelsky, P. Roushan, J. Seo, Q. Xu, H. W. Zandbergen, A. Yazdani, N. P. Ong, and R. J. Cava, Phys. Rev. Lett. 104, 057001 (2010).

[43] L. A. Wray, S.-Y. Xu, Y. Xia, Y. S. Hor, D. Qian, A. V. Fedorov, H. Lin, A. Bansil, R. J. Cava, and M. Z. Hasan, Nat. Phys. 6, 855 (2010).

[44] M. C. Diamantini, P. Sodano, and C. A. Trugenberger, New J. Phys. 14, 063013 (2012).

[45] Shruti, V. K. Maurya, P. Neha, P. Srivastava, and S. Patnaik, Phys. Rev. B 92, 020506 (2015).

[46] Z. Liu, X. Yao, J. Shao, M. Zuo, L. Pi, S. Tan, C. Zhang, and Y.Q. Zhang, J. Am. Chem. Soc. 137, 10512 (2015).

[47] H. Eyring, J. Chem. Phys. 3, 107 (1935).

[48] G. H. Vineyard, J. Phys. Chem. Solids 3, 121 (1957).

[49] P. Hänggi, P. Talkner, and M. Borkovec, Rev. Mod. Phys. 62, 251 (1990)

[50] K. J. Laidler and M. C. King, J. Phys. Chem. 87, 2657 (1983).

[51] I. Vattulainen, S. C. Ying, T. Ala-Nissila, and J. Merikoski, Phys. Rev. B 59, 7697 (1999).

[52] I. Vattulainen, J. Merikoski, T. Ala-Nissila, and S. C. Ying, Phys. Rev. B 57, 1896 (1998).

[53] P. Salo, J. Hirvonen, I. T. Koponen, O. S. Trushin, J. Heinonen, and T. Ala-Nissila, Phys. Rev. B 64, 161405 (2001). 
[54] J. R. Sanchez and J. W. Evans, Phys. Rev. B 59, 3224 (1999).

[55] U. Titulaer and J. Deutch, J. Chem. Phys. 77, 472 (1982).

[56] A. Kley, P. Ruggerone, and M. Scheffler, Phys. Rev. Lett. 79, 5278 (1997).

[57] J. Haus and K. Kehr, Phys. Rep. Rev. Sec. Phys. Lett. 150, 263 (1987).

[58] J. A. Revelli, C. E. Budde, D. Prato, and H. S. Wio, New J. Phys. 7, 16 (2005).

[59] R. Condit, in Kinetics of Reactions in Ionic Systems. Materials Science Research, edited by T. Gray and V. Fréchette (Springer, New York, 1969), pp. 284-303.

[60] R. Condit, R. Hobbins, and C. Birchenall, Oxidat. Metals 8, 409 (1974).

[61] D. Birnie, J. Phys. Chem. Solids 51, 1313 (1990).

[62] M. A. Gosalvez, M. M. Otrokov, N. Ferrando, A. G. Ryabishchenkova, A. Ayuela, P. M. Echenique, and E. V. Chulkov, Phys. Rev. B, submitted.

[63] J. G. LePage, M. Alouani, D. L. Dorsey, J. W. Wilkins, and P. E. Blöchl, Phys. Rev. B 58, 1499 (1998).

[64] C. Ratsch and M. Scheffler, Phys. Rev. B 58, 13163 (1998).
[65] H. Yildirim, A. Kara, S. Durukanoglu, and T. Rahman, Surf. Sci. 600, 484 (2006).

[66] H. Yildirim, A. Kara, and T. S. Rahman, Phys. Rev. B 76, 165421 (2007).

[67] P. E. Blöchl, Phys. Rev. B 50, 17953 (1994).

[68] G. Kresse and J. Furthmüller, Phys. Rev. B 54, 11169 (1996).

[69] G. Kresse and D. Joubert, Phys. Rev. B 59, 1758 (1999).

[70] J. P. Perdew, K. Burke, and M. Ernzerhof, Phys. Rev. Lett. 77, 3865 (1996).

[71] S. Grimme, J. Comput. Chem. 27, 1787 (2006).

[72] H. Jónsson, G. Mills, and K. W. Jacobsen, in Classical and Quantum Dynamics in Condensed Phase Simulations, edited by G. C. B. J. Berne and D. F. Coker (World Scientific, Singapore, 1998).

[73] D. Sheppard, P. Xiao, W. Chemelewski, D. D. Johnson, and G. Henkelman, J. Chem. Phys. 136, 074103 (2012).

[74] G. Henkelman and H. Jónsson, J. Chem. Phys. 111, 7010 (1999).

[75] T. Ikeda, T. Watanabe, H. Itoh, and T. Ichinokawa, Surf. Rev. Lett. 03, 1377 (1996). 